\title{
Three-dimensional visualization of ensemble weather forecasts - Part 2: Forecasting warm conveyor belt situations for aircraft-based field campaigns
}

\author{
M. Rautenhaus ${ }^{1}$, C. M. Grams ${ }^{2}$, A. Schäfler ${ }^{3}$, and R. Westermann ${ }^{1}$ \\ ${ }^{1}$ Computer Graphics \& Visualization Group, Technische Universität München, Garching, Germany \\ ${ }^{2}$ Institute for Atmospheric and Climate Science, ETH Zürich, Zurich, Switzerland \\ ${ }^{3}$ Deutsches Zentrum für Luft- und Raumfahrt, Institut für Physik der Atmosphäre, Oberpfaffenhofen, Germany \\ Correspondence to: M. Rautenhaus (marc.rautenhaus@tum.de)
}

Received: 4 February 2015 - Published in Geosci. Model Dev. Discuss.: 27 February 2015

Revised: 23 June 2015 - Accepted: 7 July 2015 - Published: 31 July 2015

\begin{abstract}
We present the application of interactive threedimensional (3-D) visualization of ensemble weather predictions to forecasting warm conveyor belt situations during aircraft-based atmospheric research campaigns. Motivated by forecast requirements of the T-NAWDEX-Falcon 2012 (THORPEX - North Atlantic Waveguide and Downstream Impact Experiment) campaign, a method to predict 3-D probabilities of the spatial occurrence of warm conveyor belts (WCBs) has been developed. Probabilities are derived from Lagrangian particle trajectories computed on the forecast wind fields of the European Centre for Medium Range Weather Forecasts (ECMWF) ensemble prediction system. Integration of the method into the 3 -D ensemble visualization tool Met.3D, introduced in the first part of this study, facilitates interactive visualization of WCB features and derived probabilities in the context of the ECMWF ensemble forecast. We investigate the sensitivity of the method with respect to trajectory seeding and grid spacing of the forecast wind field. Furthermore, we propose a visual analysis method to quantitatively analyse the contribution of ensemble members to a probability region and, thus, to assist the forecaster in interpreting the obtained probabilities. A case study, revisiting a forecast case from T-NAWDEX-Falcon, illustrates the practical application of Met.3D and demonstrates the use of 3-D and uncertainty visualization for weather forecasting and for planning flight routes in the medium forecast range (3 to 7 days before take-off).
\end{abstract}

\section{Introduction}

Weather forecasting during aircraft-based field campaigns requires the meteorologist to explore large amounts of numerical weather prediction (NWP) data in a short period of time. Atmospheric features relevant to a research flight have to be identified quickly, and findings have to be communicated to colleagues. Furthermore, assessing the forecast's uncertainty has become indispensable as flights frequently have to be planned several days before take-off.

A challenging element in forecasting methodology is to create clear and intuitive visualizations that allow the meteorologist to perform these tasks in a timely manner. To advance forecasting techniques for research flight planning, this work presents a new approach using interactive threedimensional (3-D) visualization of ensemble weather predictions (the latter a major source of information on forecast uncertainty; Gneiting and Raftery, 2005; Leutbecher and Palmer, 2008) to forecast warm conveyor belt (WCB) situations.

The article is the second part of a two-paper study. The first part (Rautenhaus et al., 2015, hereafter "Part 1") introduces "Met.3D", a tool providing interactive 3-D techniques for the visual exploration of ensemble weather prediction data. This article focuses on the specific application case of forecasting WCBs: strong, ascending and often rain producing airstreams associated with extratropical cyclones. The term "WCB" was introduced by Harrold (1973) and Browning (1971) and consolidated in a conceptual airstream model for extratropical cyclones (also including the cold conveyor 
belt and the dry airstream) by Carlson (1980). Example references for WCBs include Browning (1990) for an overview, Eckhardt et al. (2004) and Madonna et al. (2014) for a climatology, and Browning (1986) and Pfahl et al. (2014) for relevance in large-scale precipitation. WCBs are an atmospheric feature that has been in the focus of several aircraft-based campaigns (e.g. Pomroy and Thorpe, 2000; Vaughan et al., 2003; Schäfler et al., 2014; Vaughan et al., 2015).

A recent campaign that targeted WCBs is T-NAWDEXFalcon 2012 (THORPEX - North Atlantic Waveguide and Downstream Impact Experiment, hereafter TNF), which took place in October 2012 in southern Germany. Schäfler et al. (2014) described the TNF flight-planning process. WCBs (as well as other atmospheric features targeted by research flights) are of an inherently 3-D nature. However, although the atmosphere is $3-\mathrm{D}$, the forecasting and flight-planning tools employed during TNF relied on two-dimensional (2-D) visualization methods. This is a common property not only of campaign tools (Flatøy et al., 2000; Blakeslee et al., 2007; He et al., 2010; Rautenhaus et al., 2012) but also of meteorological workstations in general (e.g. Heizenrieder and Haucke, 2009; Russell et al., 2010). 3-D visualization methods are not commonly used in forecasting. While 3-D techniques have been used in research settings as early as in the 1980s (e.g. Grotjahn and Chervin, 1984; Hibbard, 1986; Hibbard et al., 1989; Wilhelmson et al., 1990) and continue to be used in recent visualization tools (e.g. Hibbard, 2005; Norton and Clyne, 2012; Dyer and Amburn, 2010; Murray and McWhirter, 2007), only few reports on approaches using 3-D techniques for forecasting have been published in the past 2 decades (Treinish and Rothfusz, 1997; Koppert et al., 1998; McCaslin et al., 2000). Part 1, Sect. 2, provides further details on the listed references and on 3-D visualization in meteorology.

Similarly, while the use of ensemble predictions has been reported for recent field campaigns (e.g. Wulfmeyer et al., 2008; Elsberry and Harr, 2008; Ducrocq et al., 2014; Vaughan et al., 2015), they have, to the best of our knowledge, not been used to create specific 3-D forecast products for flight planning. However, in particular the possibility to use ensembles to compute 3-D probability fields of the occurrence of features or events is valuable for flight planning. For the WCB case, a probability of WCB occurrence can be used to plan flight routes in regions in which the probability to encounter a WCB is at a maximum.

The work presented in this article is motivated by the questions of (1) how interactive 3-D visualization can be used to improve the exploration of 3-D features of interest to a flight campaign, and (2) how ensemble forecasts (in particular derived probabilities) can be used to improve research flight planning in the medium forecast range (that is, three to seven days before take-off). Our developments have been guided by a number of forecast questions that reflect the TNF requirements. They are repeated here from Part 1 for completeness:
A. How will the large-scale weather situation develop over the next week, and will conditions occur that favour WCB formation?

B. How uncertain are the weather predictions?

C. Where and when, in the medium forecast range and within the spatial range of the aircraft, is a WCB most likely to occur?

D. How meaningful is the forecast of WCB occurrence?

E. Where will the WCB be located relative to cyclonic and dynamic features?

The technical basis for questions A and B is laid in Part 1. This article addresses questions $\mathrm{C}$ to $\mathrm{E}$ and presents a case study that demonstrates how the methods developed in both papers are applied to forecasting.

The paper is structured as follows. In Sect. 2, we propose a technique to compute 3-D probabilities of WCB occurrence. Our approach is put into relation to previous work in the field, and its integration into the Met.3D architecture is described. During TNF, we followed the approach of Wernli and Davies (1997) and used Lagrangian particle trajectories computed on the forecast wind field to objectively detect WCB airstreams. Using wind forecasts from the European Centre for Medium Range Weather Forecasts (ECMWF) Ensemble Prediction System (ENS; comprising 50 perturbed forecast runs and an unperturbed control run; e.g. Buizza et al., 2006), trajectories were started from the atmospheric boundary layer (ABL) for each ensemble member. Those trajectories fulfilling a WCB criterion were gridded into 2-D grids and displayed as probability maps showing the occurrence of either or all of WCB inflow, ascent and outflow. However, generalising this approach to three dimensions poses challenges, as discussed in Sect. 2. We present an adapted approach using domain-filling trajectories, which is more accurate, albeit computationally more expensive. In order to find the best method that is still computationally tractable in a forecast setting, both approaches are compared in Sect. 3. We analyse their sensitivity to the grid spacing of the forecast wind fields and to the number and locations of the trajectory seeding points.

To facilitate quantitative interpretation of the obtained probabilities, we further propose a visual analysis method for cases in which only low probabilities of the occurrence of WCBs are encountered (Sect. 4). In such cases a flight often might not be planned due to the interpreted high uncertainty. However, low probability can have two causes. Either indeed only a small percentage of the ensemble members predict a WCB feature, or large spatial variation of the features in the individual ensemble members causes only marginal overlap and thus low probabilities. In the latter case the probability that a WCB will occur is actually much larger than suggested by the visualized probabilities. However, there is a large uncertainty in where it will occur. To help the user distinguish 
between these causes, we propose a method that identifies the contribution of individual members to a probability region.

After the introduction of all methods that are required to explore a forecast to answer forecast questions A to E, Sect. 5 revisits the TNF forecast case of 19 October 2012. The case study shows how the proposed 3-D ensemble visualization workflow is applied to campaign forecasting, and illustrates the use and added value of the presented methods.

The paper is concluded with a summary and discussion in Sect. 6.

\section{Probability of warm conveyor belt occurrence}

WCBs are Lagrangian airstreams in extratropical cyclones (e.g. Harrold, 1973; Carlson, 1980; Browning, 1990). They transport warm and moist air from the ABL in a cyclone's warm sector upward and poleward towards the tropopause. The inflow region in the lower troposphere typically extends over several hundred kilometres in diameter. WCB air masses commonly ascend by about $500-600 \mathrm{hPa}$ in $48 \mathrm{~h}$, thereby covering horizontal distances of up to $2000 \mathrm{~km}$ (e.g. Wernli and Davies, 1997; Eckhardt et al., 2004). Due to the strong ascent, condensation leads to strong latent heat release and the formation of clouds and precipitation (e.g. Browning, 1986). Therefore, WCBs are highly relevant for precipitation extremes in the extratropics (e.g. Pfahl et al., 2014). Once the air masses reach jet level, an outflow region forms near the tropopause. This region is characterized by cirrus clouds that extend over several thousand kilometres along the jet stream. Readers interested in further detail are referred to Madonna et al. (2014), who give a comprehensive introduction to the field.

To plan a flight that allows for aircraft measurements within a WCB, we are interested in the spatial and temporal distribution of $\mathrm{WCB}$ features in the ensemble forecast. As a summary measure of the uncertainty information, the probability of WCB occurrence, $p(\mathrm{WCB})$, is of particular interest. It provides for a given location in 3-D space at a given time the probability of encountering a WCB air mass. To compute $p(\mathrm{WCB})$ from an ensemble weather forecast, we first need to detect WCB features in the individual ensemble members.

\subsection{WCB detection based on objectively selected Lagrangian particle trajectories}

In early studies of, for instance, Harrold (1973), Carlson (1980) and Browning (1986), conveyor belt airstreams have been identified by manual inspection of satellite imagery or by isentropic analysis. Subsequent studies have used Lagrangian particle trajectories computed with wind fields from numerical model output to investigate case studies of extratropical cyclones. For example, Whitaker et al. (1988) and Hibbard et al. (1989) show the existence of three distinct airstreams in a modelling case study of the 1979 "President's Day storm" and relate the airstreams to the conceptual model by Carlson (1980). Further case studies, including Kuo et al. (1992), Schultz and Mass (1993), Mass and Schultz (1993) and Reed et al. (1994), also interpret computed trajectories in consideration of the Carlson (1980) model; however, note that they are able to identify rather a continuum of flow paths than discrete airstreams.

In more recent studies (see discussion below), Lagrangian particle trajectories are frequently used to objectively detect WCB structures in numerical model output. For our work, we are interested in the specific ways trajectories are used in the literature to detect WCBs. In particular, this includes the employed objective detection criteria and the spatial and temporal spacing of the trajectories as well as the employed wind fields.

Wernli and Davies (1997) introduced objective criteria to extract what they call "coherent ensembles of trajectories" (CET; a bundle of trajectories started at different locations; not to be confused with the meaning of "ensemble" in "ensemble forecasts") from a set of trajectories covering the entire domain of interest. They use wind fields from the ECMWF global atmospheric model, interpolated (from a spectral truncation of T213) to a regular latitude-longitude grid of $0.75^{\circ} \times 0.75^{\circ}$ with 31 levels in the vertical and a $6 \mathrm{~h}$ time interval. Trajectories are started on every model grid point below $800 \mathrm{hPa}$ (approx. seven levels). Wernli and Davies show that nearly identical CETs are obtained by selecting trajectories that experience either a moisture decrease of $12 \mathrm{~g} \mathrm{~kg}^{-1}$ in $48 \mathrm{~h}$ or an ascent of more than $620 \mathrm{hPa}$ in $48 \mathrm{~h}$. The approach allows one to focus on the dynamically most relevant cores of an extratropical cyclone's airstreams. In a subsequent article, Wernli (1997) applied the suggested method to the case study of Browning and Roberts (1994) and relates the obtained CETs to the WCB model. Unlike the analysis of a continuum of airstreams in a cyclone, this method selects the strongest ascending air masses within the WCB.

Stohl (2001) and Eckhardt et al. (2004) computed climatologies of WCBs. Stohl (2001) seeded the trajectories on a $1^{\circ} \times 1^{\circ}$ grid in the horizontal and on two vertical levels at 500 and 1500 ma.s.l. (above sea level). He noted that the results of his climatology are sensitive to the WCB selection criterion, and settled for the - as he writes - "somewhat arbitrary" criterion of $8000 \mathrm{~m}$ in $48 \mathrm{~h}$ (the approximate timescale at which air flows through a single synoptic system). Similarly, Eckhardt et al. (2004) started trajectories on a $1^{\circ} \times 1^{\circ}$ grid at $500 \mathrm{ma.s} .1$. They noted that "any criterion used for an automatic classification of WCBs is necessarily subjective". In their work, trajectories travelling more than $10^{\circ}$ eastward and $5^{\circ}$ northward and ascending more than $60 \%$ of the average tropopause height within $48 \mathrm{~h}$ are classified as WCB trajectories.

A number of studies use the trajectory model "Lagrangian analysis tool" (LAGRANTO) (Sprenger and 
Wernli, 2015), originally introduced by Wernli and Davies (1997). Spichtinger et al. (2005) analysed ice supersaturation in the vicinity of a WCB's outflow region, Grams et al. (2011) presented a case study of an extratropical transition. Schäfler et al. (2011) analysed aircraft measurements and Madonna et al. (2014) presented a climatology of WCBs. All four studies settle for a criterion of an ascent of more than $600 \mathrm{hPa}$ in $48 \mathrm{~h}$ to select WCB trajectories. In terms of seeding, Schäfler et al. (2011) started their trajectories on every model grid point between the surface and $850 \mathrm{hPa}$ of the deterministic ECMWF T799L91 forecast (spectral truncation of T799, with 91 vertical levels), interpolated to a regular latitude-longitude grid of $0.25^{\circ} \times 0.25^{\circ}$, and using the approximately 17 lowest levels. Madonna et al. (2014) seed their trajectories at $80 \mathrm{~km}$ distance in the horizontal and at $20 \mathrm{hPa}$ vertical distance on levels between 1050 and $790 \mathrm{hPa}$. Their wind field is available at $1^{\circ} \times 1^{\circ}$ grid spacing.

During TNF (Schäfler et al., 2014), LAGRANTO has been used with wind fields from the ECMWF ensemble forecast covering the North Atlantic and Europe. To keep the computational demand tractable for the operational forecast setting, the available ENS spectral resolution of T639 was interpolated to $1^{\circ} \times 1^{\circ}$ in latitude and longitude. In the vertical, all available 62 levels were used. A $6 \mathrm{~h}$ time step was used. Trajectories were started for each member at $1^{\circ}$ horizontal spacing at five levels constant in pressure between 1000 and $800 \mathrm{hPa}$. The selection criterion was set to an ascent of $500 \mathrm{hPa}$ in $48 \mathrm{~h}$.

In summary, the reviewed studies have all restricted trajectory seeding to lower atmospheric levels. The horizontal distance between start points mostly corresponds to the grid spacing of the driving wind fields. While the exact selection criterion for WCB trajectories varies, all studies use a criterion that filters trajectories according to a given ascent in a two day period.

\subsection{Computation of $p(\mathrm{WCB})$}

We follow the approach of Wernli and Davies (1997) and detect WCB features by selecting Lagrangian particle trajectories according to a given ascent $\Delta p$ in a given time period $\Delta t$. Trajectories are computed with LAGRANTO. We use the same ECMWF ENS wind fields described in detail in Part 1, Sect. 4.1. From the available spectral truncation of T639, the wind forecasts are horizontally interpolated by the ECMWF Meteorological Archive and Retrieval System (MARS) to a regular latitude-longitude grid of $1^{\circ} \times 1^{\circ}$ (the same data used during TNF) and (additionally) $0.25^{\circ} \times 0.25^{\circ}$. In the vertical, the ECMWF model uses hybrid sigma-pressure coordinates (Untch and Hortal, 2004, also cf. Fig. 9 in Part 1), of which all available 62 model levels are used.

Once trajectories have been computed and selected, a gridded field of $p(\mathrm{WCB})$ can be derived by relating each ensemble member's trajectories to a binary grid, and by computing for each grid point the relative number of members that pre- dict a WCB feature at that grid point. In a more formal way, the method to compute $p$ (WCB) at time $t$ can be summarized as follows:

1. For every ensemble member $m$ and every available forecast time step $t_{0} \in(t-48 \mathrm{~h} . . t)$, integrate 3-D Lagrangian particle trajectories, started at a fixed set of seeding points, from $t_{0}$ forward in time for $\Delta t=48 \mathrm{~h}$.

2. Select those trajectories that fulfil a specified WCB criterion (e.g. an ascent of $\Delta p=600 \mathrm{hPa}$ in $\Delta t=48 \mathrm{~h}$ ).

3. For each member $m$, create a 3-D binary grid $B^{m}$ that for every grid point with indices $k, j, i, B_{k j i}^{m}$, contains a set bit $\left(B_{k j i}^{m}=1\right)$ if the grid point is located "inside" a WCB air mass at time $t$, where "inside" needs to be determined from the trajectory positions at $t$.

4. For each grid point compute the probability of WCB occurrence by counting the number of members with a set bit for the point: $p(\mathrm{WCB})_{k j i}=1 / M \sum_{m} B_{k j i}^{m}$, where $M$ denotes the number of ensemble members.

For trajectories seeded approximately in the atmospheric boundary layer, we call this method an "ABL-T method". Note that the grid topology of $B$ needs to be identical for each member in order to avoid errors due to variations in grid point positions, as is the case for probabilities derived from ECMWF NWP output (cf. Sect. 5 in Part 1).

The method poses several challenges. With respect to step (1), trajectory seeding needs to be sufficiently dense to spatially sample the WCB features. The literature reviewed in Sect. 2.1 indicates that grid spacings of $1^{\circ}$ or less should be sufficient. For step (2), the WCB criterion must be carefully chosen, as the ascent that a trajectory experiences may depend on factors including seasonal variability or the horizontal and vertical grid spacing of the employed wind forecasts. Also, interactivity must be considered to enable a user to change $\Delta p$ and $\Delta t$ during forecasting to judge the sensitivity of $p$ (WCB) on these parameters. Third, we need to find a suitable gridding strategy that determines in step (3) whether a grid point is located inside a WCB air mass. The simplest approach is to extract, for each member, the particle positions of all WCB trajectories at time $t$, and to compute for each particle the grid cell $B_{k j i}^{m}$ in which it is contained.

During TNF, this simple approach was applied in 2-D to compute $p(\mathrm{WCB})$ for total grid columns, as well as for three vertical intervals to distinguish inflow, ascent and outflow. In the horizontal, a regular grid with a spacing of $1^{\circ} \times 1^{\circ}$ in latitude and longitude was used. However, with this approach no physical assumptions are made about the air volume represented by each particle. The result is sensitive to both trajectory seeding points and grid topology of $B$, and the positions of the WCB particles are only captured with an accuracy of the order of the grid spacing of $B$. Also, due to the changing area of the grid cells with latitude, the result is biased towards 


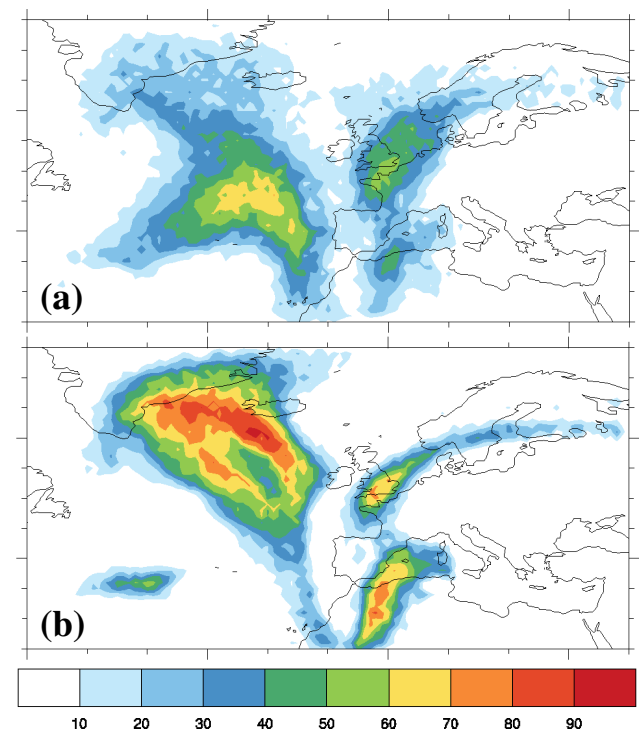

Figure 1. Total column probability of WCB occurrence (\%), as available during TNF. Probabilities are computed from ABL-started trajectories filtered for an ascent of $500 \mathrm{hPa}$ in $48 \mathrm{~h}$. Forecasts from (a) 00:00 UTC, 15 October 2012 and from (b) 00:00 UTC, 17 October 2012, both valid at 18:00 UTC, 19 October 2012. Compare to Fig. 3 in Schäfler et al. (2014).

lower probabilities close to the poles. Examples of the resulting total column $p$ (WCB) field are shown in Fig. 1 and can also been found in Schäfler et al. (2014, their Fig. 3). Due to the described issues, the results should only be interpreted in a qualitative manner.

In 3-D, more complexity is added as the vertical extent of the grid cells also has to be taken into account. To eliminate bias and sensitivity, one possibility is to assume an air parcel mass and geometry for the trajectory particles, as illustrated in Fig. 2a. In the example, the particle is associated with a spherical air parcel. Given the required thermodynamic variables at the particle position at start time $t_{0}$ and gridding time $t$, the volume and thus radius of the parcel at $t$ can be computed and the overlapping grid points found. However, due to the large difference in vertical and horizontal scale of our grids (of the order of $100 \mathrm{~km}$ in the horizontal and $100 \mathrm{~m}$ in the vertical), the usage of spherical geometry requires the computation of a very large number of trajectories. Yet, geometry that reflects the different scales (for example ellipses, cylinders or simple rectangular boxes) is difficult to motivate physically. Also, usage of large air parcels neglects potential deformation of the parcels by the wind field.

An approach not requiring any such assumptions is to use domain-filling trajectories (in the following referred to as "DF-T method"). Here, we first specify the grid topology for $B$. Next, as illustrated in Figs. 2b, c and 3, for every member and each grid point $B_{k j i}^{m}$, a trajectory starting on $B_{k j i}^{m}$ is computed. This way, we can be certain that each $B_{k j i}^{m}$ is placed exactly on a trajectory and no assumptions about the shape of the particle volume need to be made. After applying a WCB selection criterion to the trajectories, the bits of the grid points from which WCB trajectories were started are set. However, the approach requires increased computational resources. Seeding points are now required on all tropospheric layers and hence a larger number of trajectories is required. Also, trajectories additionally have to be computed backward in time to also capture those situations in which a WCB trajectory passes its seeding point in the ascent or outflow phase. Step (1) in the method description above is hence extended to also integrate the trajectories backward in time for $\Delta t$ hours from time $t$.

As an example, Fig. 3 shows results of selecting domainfilling trajectories that ascend more than $500 \mathrm{hPa}$ in $48 \mathrm{~h}$ (Fig. 3a-c) and more than $600 \mathrm{hPa}$ in $48 \mathrm{~h}$ (Fig. 3d-f). Note how the $30 \%$ isosurface of $p$ (WCB) over the English Channel almost vanishes with $600 \mathrm{hPa}$ filtering (Fig. 3f).

In Sect. 3, we compare four DF-T and ABL-T set-ups with varying grid topology with respect to obtained $p(\mathrm{WCB})$ and to computational demand. The comparison allows to find a set-up well-suited for usage in campaign forecasting.

\subsection{Implementation}

Trajectories computed with LAGRANTO are stored in NetCDF files. Trajectory selection and the computation of $p$ (WCB) take place in Met.3D and have been implemented in a number of modules in the Met.3D data processing pipeline described in Part 1, Sect. 4.2. Analogous to Fig. 10 in Part 1, Fig. 4 shows an example set-up. Separate pipeline modules are responsible for reading trajectory data from disk, filtering the data according to the selection criterion, gridding and probability computation. This architecture allows modules to be exchanged when, for example, data from a different trajectory model should be read or a different selection criterion should be applied.

Hardware permitting, parts of the pipeline (for example, trajectory selection) can be executed in parallel. Intermediate results in the pipeline are cached by a memory manager. Both parallel execution and caching increase the interactivity of the system with respect to changing the selection parameters $\Delta p$ and $\Delta t$. For further details on the Met.3D pipeline architecture, we refer the reader to Part 1, Sect. 4.2.

To select trajectories according to the ascent criterion, the maximum pressure change occurring within a trajectory over the time interval $\Delta t$ is required. For the grid spacings used here, the data volume of the trajectories of all members amounts to multiple GB per time step if stored in binary NetCDF format (approximately $2.4 \mathrm{~GB}$ for $1^{\circ}$ horizontal grid spacing if a vertical region of interest of 52 levels is selected, and approximately $38 \mathrm{~GB}$ if the horizontal grid spacing is decreased to $0.25^{\circ}$ ). Reading the data from disk and performing the selection can hence be slow. We thus make use of the fact that the only information required to compute the probabilities is whether the trajectory started from a grid point 

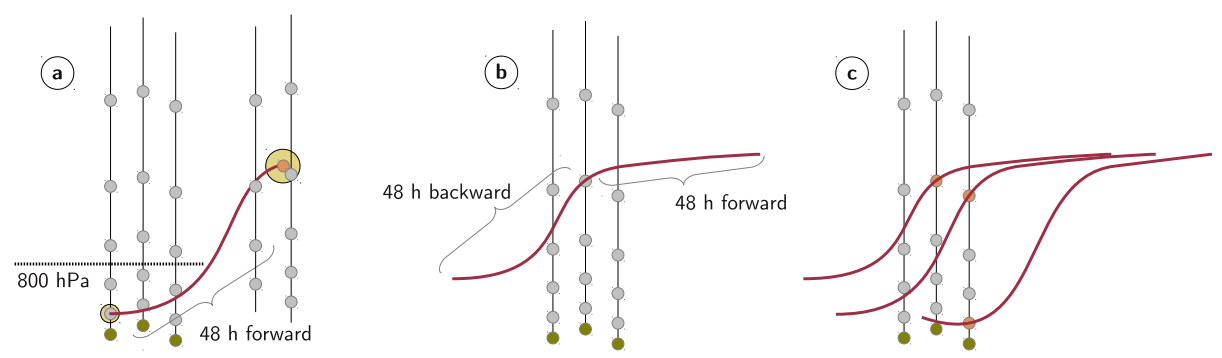

Figure 2. Methods to compute $p(\mathrm{WCB})$. (a) ABL-T method using trajectories started in the atmospheric boundary layer and integrated $48 \mathrm{~h}$ forward in time. To get 3-D gridded information on WCB location, an air parcel volume needs to be assumed for each particle so that grid points overlapping with the volume can be determined. (b) DF-T method using domain-filling trajectories started from every grid point of the $p(\mathrm{WCB})$ grid and integrated both $48 \mathrm{~h}$ forward and backward in time. No volume has to be assumed as selected WCB trajectories are located exactly on grid points (c).
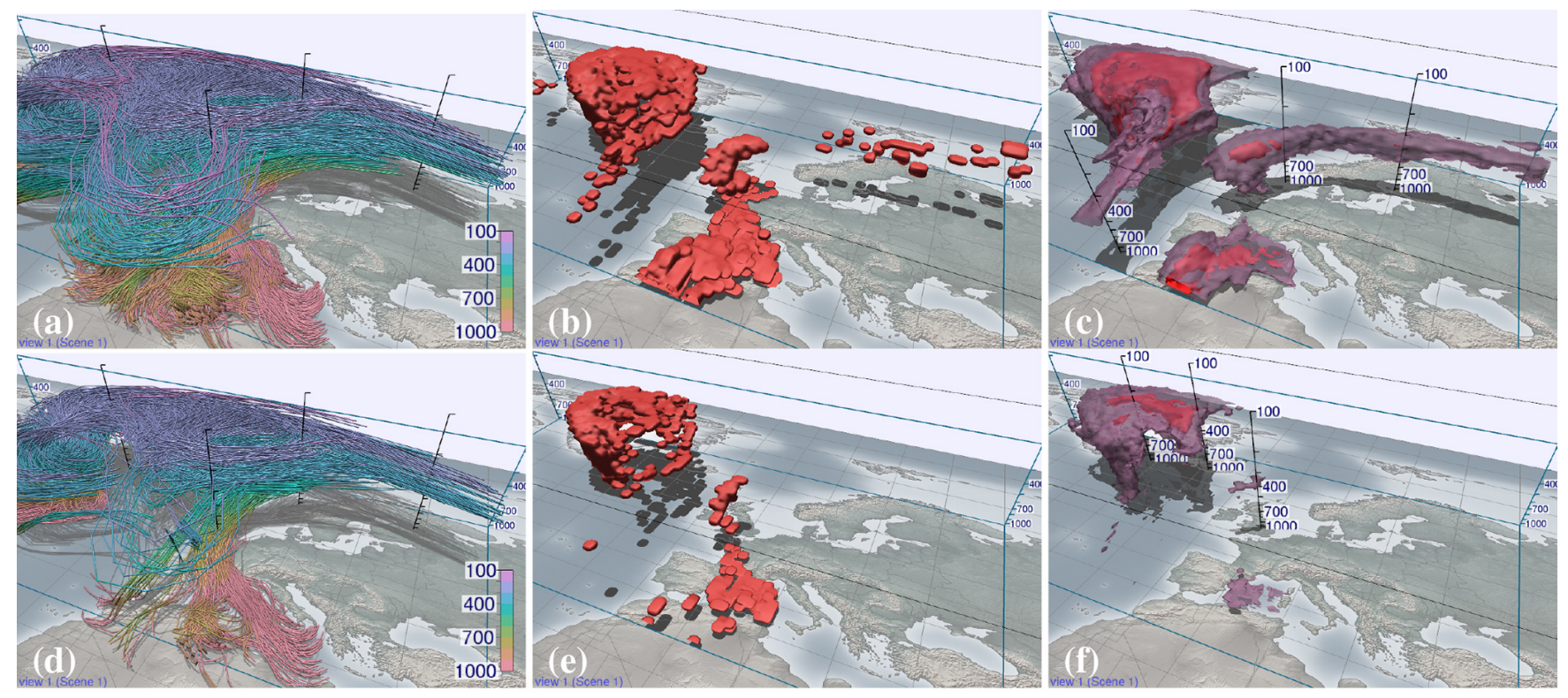

Figure 3. Derivation of $p(\mathrm{WCB})$ with DF-T set-up (S3). (a, d) Trajectories started at 18:00 UTC, 19 October 2012, computed with wind fields from the ensemble control forecast from 00:00 UTC, 17 October 2012, integrated forward and backward in time for $48 \mathrm{~h}$ each. Trajectories are selected according to an ascent of (a-c) 500 and (d-f) $600 \mathrm{hPa}$ in $48 \mathrm{~h}$. Colour encodes altitude (hPa). (b, e) Volume rendering of the binary grid $B$, representing the start positions of the selected trajectories. (c, f) Probability of WCB occurrence derived from all 51 members of the ensemble. The red opaque isosurface shows $30 \%$ probability, the purple transparent isosurface $10 \%$ probability. Vertical axes are labelled with pressure altitude $(\mathrm{hPa})$.

fulfils the selection criterion. The data volume that needs to be loaded can be largely reduced by pre-computing the maximum pressure change $\Delta p$ for a range of time intervals $\Delta t$. Now, for a given $\Delta t$, only the maximum $\Delta p$ for each trajectory (i.e. grid point) needs to be read. The selection process is reduced to comparing each trajectory's $\Delta p$ to the given threshold value. This way, we are able to provide an interactively adjustable selection criterion to the user.

\section{Choice of $p(\mathrm{WCB})$ method and grid spacing for forecasting}

To use a $p(\mathrm{WCB})$ method for forecasting during a campaign, a number of criteria need to be fulfilled:

a. The trajectories need to be computed in a short period of time (for our application this is preferably less than one hour), so that results are available soon after the forecast wind fields become available from ECMWF (with "available" we mean that the retrieval of the forecast data from MARS, yielding the interpolated wind fields described in Part 1, Sect. 4.1, has finished); 


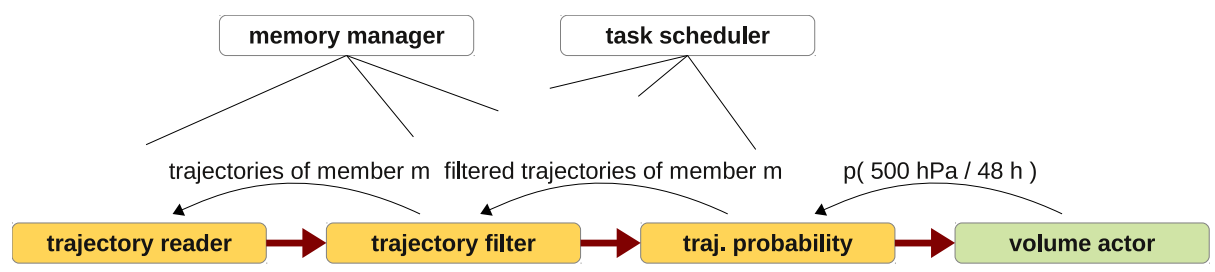

Figure 4. Sample Met.3D data processing pipeline to visualize $p(\mathrm{WCB})$, depicted analogous to Fig. 10 in Part 1 . Different pipeline modules (yellow) are responsible for reading and selecting trajectories, and for computing the $p$ (WCB) field. In the example, a volume actor (green; for details see Part 1, Sect. 3) visualizes the resulting $p(\mathrm{WCB})$ data. A request for the probability of occurrence of trajectories, emitted by the volume actor, triggers further requests up the pipeline. Intermediate results are cached by the memory manger, connected to each pipeline module (indicated by the black lines). Pipeline execution can be parallel and is controlled by a task scheduler, also connected to all pipeline modules (for details see Part 1, Sect. 4.2).

b. the amount of trajectory data needs to be small enough to be handled interactively in Met.3D;

c. the grid spacing needs to be fine enough to capture the important features that are present in a "best-possible" forecast.

\subsection{Evaluated set-ups}

We evaluate four different set-ups with respect to the given criteria:

S1. As the "best-possible" $p(\mathrm{WCB})$ forecast, we use a DF$T$ set-up with trajectories computed on the ECMWF ENS wind fields at the highest available grid spacing (T639L62 spectral resolution, horizontally interpolated by MARS to a regular grid of $0.25^{\circ} \times 0.25^{\circ}$ in latitude and longitude, with 62 hybrid sigma-pressure levels in the vertical). Care must be taken with respect to the choice of the $B^{m}$ and $p$ (WCB) grids. A straightforward choice is to use the ECMWF grid on which the wind fields are available. However, the vertical position of the grid points on all but the uppermost hybrid sigma-pressure levels depends on the surface pressure field (Untch and Hortal, 2004, also cf. Part 1, Sect. 4.1), which varies between ensemble members and time steps. Hence, if for a given time step the individual members' wind grids are used for the $B^{m}$, the problem described in Part 1, Sect. 5, arises: the grid points are located at different vertical positions across the ensemble, and hence an error is introduced when computing the probability. To avoid this problem while staying as close as possible to the ECMWF grid, we use the grid defined by the time step's ensemble minimum surface pressure for the $B^{m}$ of all members. The minimum surface pressure is chosen to ensure that all grid points are located above the surface (if the mean surface pressure is used, grid points in the lowest levels can be located below the surface in some members). We focus on a vertical region of interest of up to approximately $100 \mathrm{hPa}$ and for the $B^{m}$ and $p$ (WCB) grids discard the model levels above this elevation (retaining the lower 52 levels).
S2. The same set-up as (S1), but with horizontal wind field, $B$, and $p(\mathrm{WCB})$ grid spacing reduced to $1^{\circ} \times 1^{\circ}$. As in (S1), the lower 52 vertical levels are used for $B$ and $p(\mathrm{WCB})$.

S3. The same set-up as (S2), but with $B$ and $p(\mathrm{WCB})$ grids defined by a constant surface pressure of $1000 \mathrm{hPa}$, not by the ensemble minimum surface pressure. The wind forecast data remain as in (S2). The advantage of this set-up is that the $p(\mathrm{WCB})$ grid can be interpreted as a structured pressure level grid and thus be visualized much more efficiently (Part 1, Sect. 4.3). This way, the interactivity in Met.3D can be improved. The drawback, however, is that some of the lower-level grid points are now located below the surface and become invalid. This reduces the vertical grid spacing in the lower troposphere above mountainous terrain.

S4. An ABL-T set-up using a grid $B$ that is regular in the horizontal with a grid spacing of $1^{\circ} \times 1^{\circ}$ as in (S2) and (S3). In the vertical, the grid is regular in pressure with a grid spacing of $10 \mathrm{hPa}$. This spacing is of the order of the average spacing of the model level grids used in (S2) and (S3), and results in a comparable number of vertical levels in the region of interest (90 levels between 1000 and $100 \mathrm{hPa}$ ). Usage of a regular pressure level grid can be motivated physically; from hydrostatic balance (e.g. Wallace and Hobbs, 2006, Sect. 3.2), we know that for a column of air with constant mass $m$ the difference in pressure $\delta p$ between top and bottom boundary of the column stays constant with height: $-\delta p=g \rho \delta z=m g A$, where $g$ is the acceleration due to gravity (assumed to be constant), $\rho$ the density of the air, $\delta z$ the geometric height of the column and $A$ the cross sectional area of the column. We start the trajectories on those grid points of $B$ that are located below $700 \mathrm{hPa}$ and classify a grid point as belonging to a WCB if a particle is positioned in the corresponding grid cell. This way, while we implicitly assume a particle geometry that is rectangular in longitude, latitude and pressure, the mass represented by the particle remains constant 
when rising at constant latitude. The artefact of decreasing grid cell area $A$ towards the poles remains, though. For trajectory integration, the same forecast data as in (S2) and (S3) are used.

For all trajectory computations, LAGRANTO is driven with ECMWF ENS forecast data at $6 \mathrm{~h}$ time steps. The model internally uses a $30 \mathrm{~min}$ time step for the integration, trajectory positions are output at $6 \mathrm{~h}$ intervals.

\subsection{Set-up comparison}

In terms of computational resources, set-up (S1) is the most demanding configuration. On our test system (six-core Intel Xeon running at $2.67 \mathrm{GHz} ; 24 \mathrm{~GB}$ RAM; $512 \mathrm{~GB}$ solid state drive), the computation of the trajectories of a single time step takes about $50 \mathrm{CPU}$ minutes per member. The data output for a time step of all members, stored in binary NetCDF format, amounts to approximately $38 \mathrm{~GB}$. While such simulations are feasible for research settings, they are not suited for forecasting. For set-ups (S2) and (S3), the number of trajectories decrease by a factor of 16 . The time required to compute the trajectories reduces to about $3 \mathrm{CPU}$ minutes per time step and member, about $2.4 \mathrm{~GB}$ of trajectory data are produced per time step for the entire ensemble. With the current ENS size of 51 members, this setting is feasible for forecasting if a small compute cluster is available. For setup (S4), the time further reduces to about $1 \mathrm{CPU}$ minute and data volume reduces to approximately one GB.

In Figs. 5 and 6, the four set-ups are compared by means of four typical visualizations of the Met.3D workflow: (a) the volume rendering of $p(\mathrm{WCB})$ isosurfaces already used in Fig. 3c, (b) a volume rendering of WCB features in forecast member 12 (as captured by the binary grid $B^{12}$ ), (c) a horizontal section at $410 \mathrm{hPa}$ through the ascent region associated with precipitation and (d) a horizontal section through the inflow region at $950 \mathrm{hPa}$. The TNF forecast case of $19 \mathrm{Oc}-$ tober 2012 that already served for the examples in Part 1 is used. The main features (cf. Fig. 1 in Part 1: inflow over the Mediterranean Sea, ascent over the English Channel and southern England, outflow over Scandinavia and Russia, as well as a strong ascent associated with former Hurricane Rafael over the North Atlantic) are well represented by all set-ups. However, in the regions of maximum $p$ (WCB), setups (S2) and (S3) predict probabilities that are decreased by about $10 \%$ compared to the "reference" set-up (S1). This is visible in the smaller extent of the $30 \%$ isosurface in Fig. 5a as well as in the horizontal sections (Fig. 6c, d). Also, single member WCB structures are more solid in set-up (S1), as illustrated in the 3-D view of the binary volume of member 12 (Fig. 5b). The decrease is caused by the lower horizontal grid spacing of the driving wind fields, in which fewer trajectories experience strong ascent - potentially due to smoothed vertical velocities. Nevertheless, set-ups (S2) and (S3) cap- ture the shape and location of the $p(\mathrm{WCB})$ features equally well as (S1).

The differences between set-ups (S2) and (S3) are negligible. While virtually no differences can be found in the visualizations of the WCB ascent at $410 \mathrm{hPa}$ (Fig. 6c), the differences become more pronounced in the lower atmospheric layers (Fig. 6d). This can be explained with the grid topology: at higher altitudes, the elevation of the model levels becomes increasingly independent of surface pressure (cf. Part 1 , Sect. 4.1) and hence the difference in the $p(\mathrm{WCB})$ grids vanishes. However, even at low altitudes the observed differences in $p(\mathrm{WCB})$ remain within a few percent.

The bottom rows of Figs. 5 and 6 show the results for the ABL-T set-up (S4). Despite the crude assumption with respect to air parcel geometry, the major $p(\mathrm{WCB})$ features are captured well. However, this set-up tends to predict slightly higher probabilities compared to (S2) and (S3) in the atmospheric boundary layer, and slightly lower probabilities at higher altitudes.

Results for other time steps are similar (not shown). We conclude that from the presented candidates, set-ups (S3) and (S4) are best suited to be used in a forecast setting. While showing small differences with respect to the absolute predicted values, both capture the shape and locations of regions of elevated $p(\mathrm{WCB})$. Also, both are feasible to compute in less than an hour and the results can, due to the structured vertical grid layout, be visualized more efficiently than the results computed by the set-ups based on hybrid sigmapressure vertical coordinates (cf. Part 1, Table 2) ${ }^{1}$.

\section{Probability region contribution}

The methods introduced so far allow one to visualize the computed $p$ (WCB) fields and to find regions in which the occurrence of a WCB is most likely. However, it remains an open question how the magnitudes of the displayed probabilities should be interpreted. A distinct property of the examples presented in Sect. 3 are relatively low probabilities. For instance, in Fig. 3c maximum values only reach about $30 \%$. As mentioned in the introduction, such low magnitudes can have two causes: either indeed only $30 \%$ of all ensemble members predict the WCB event, or large spatial variation of the features in the individual members causes only marginal overlap and thus low probabilities. Also, noise in the individual binary volumes can cause empty grid cells in the features and decrease probability values. Interpreting the data correctly and being able to distinguish between these

\footnotetext{
${ }^{1}$ To provide an order of magnitude of the rendering times, using the same hardware set-up as in Part 1, Table 1 (Nvidia GeForce GTX 560Ti graphics card with 2 GB of video memory on a six-core Intel Xeon running at $2.67 \mathrm{GHz}$ ) and a sampling step size of 0.1 , the isosurface visualizations in Fig. 5a require on average $361 \mathrm{~ms}$ for set-up (S2) and $102 \mathrm{~ms}$ for (S3).
} 

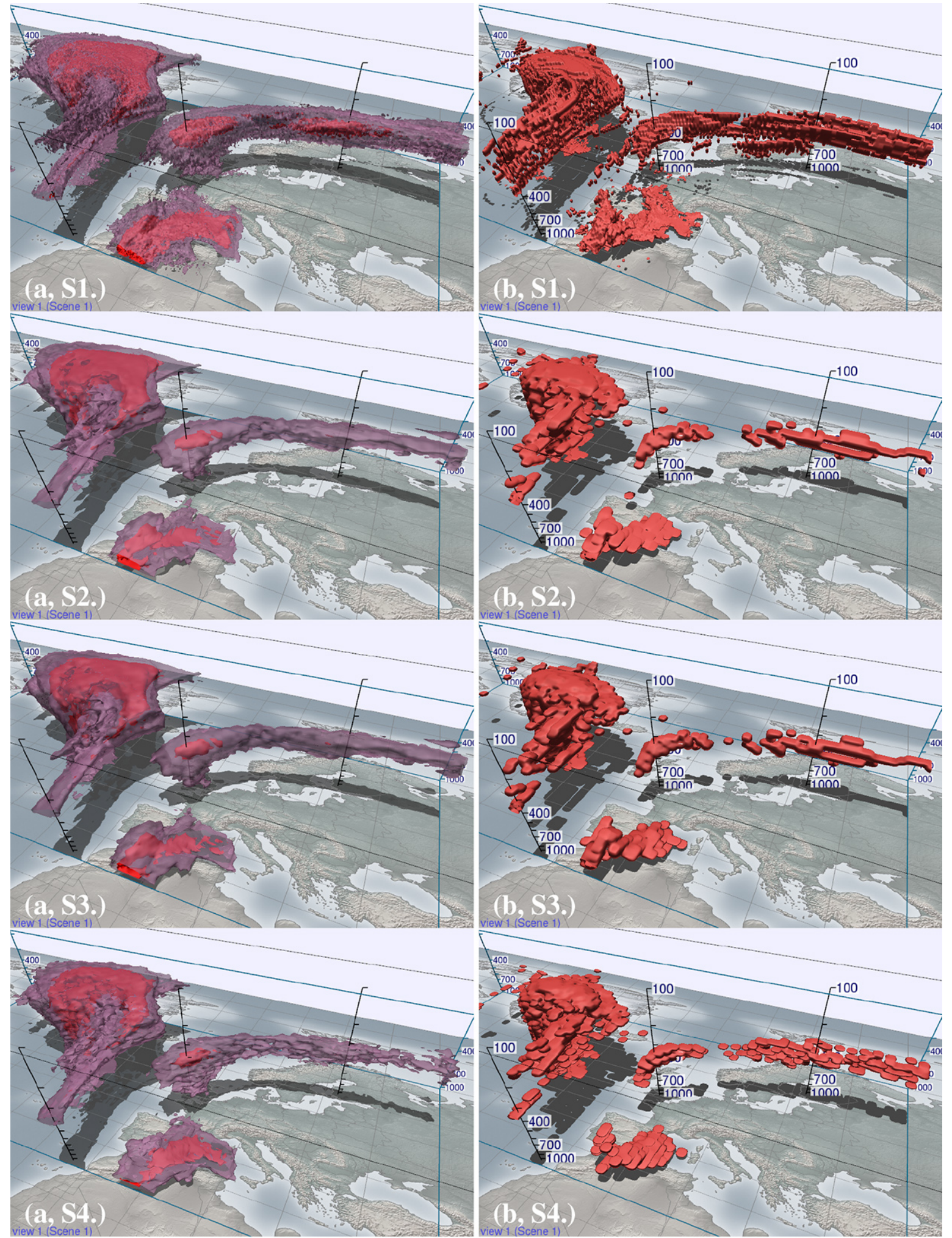

Figure 5. Comparison of set-ups to compute $p(\mathrm{WCB})$. Same forecast as in Fig. 3. The selection criterion is set to $500 \mathrm{hPa}$ in $48 \mathrm{~h}$. (a) Volume rendering of $p(\mathrm{WCB})$ (red opaque isosurface shows $30 \%$ probability, purple transparent isosurface $10 \%$ probability). (b) Volume rendering of the binary grid $B$ of a single member (member 12), representing the WCB features for this member. (S1) DF-T set-up with a horizontal grid spacing of $0.25^{\circ} \times 0.25^{\circ}$, in the vertical the lower 52 (of 62) hybrid sigma-pressure levels (up to approximately $100 \mathrm{hPa}$ ) defined by the ensemble minimum surface pressure are used. (S2) As (S1) but with $1^{\circ} \times 1^{\circ}$ horizontal grid spacing. (S3) As (S2) but with vertical levels defined by a constant surface pressure of $1000 \mathrm{hPa}$. (S4) ABL-T set-up with $1^{\circ} \times 1^{\circ}$ horizontal grid spacing and a regular vertical grid with a grid spacing of $10 \mathrm{hPa}$. 


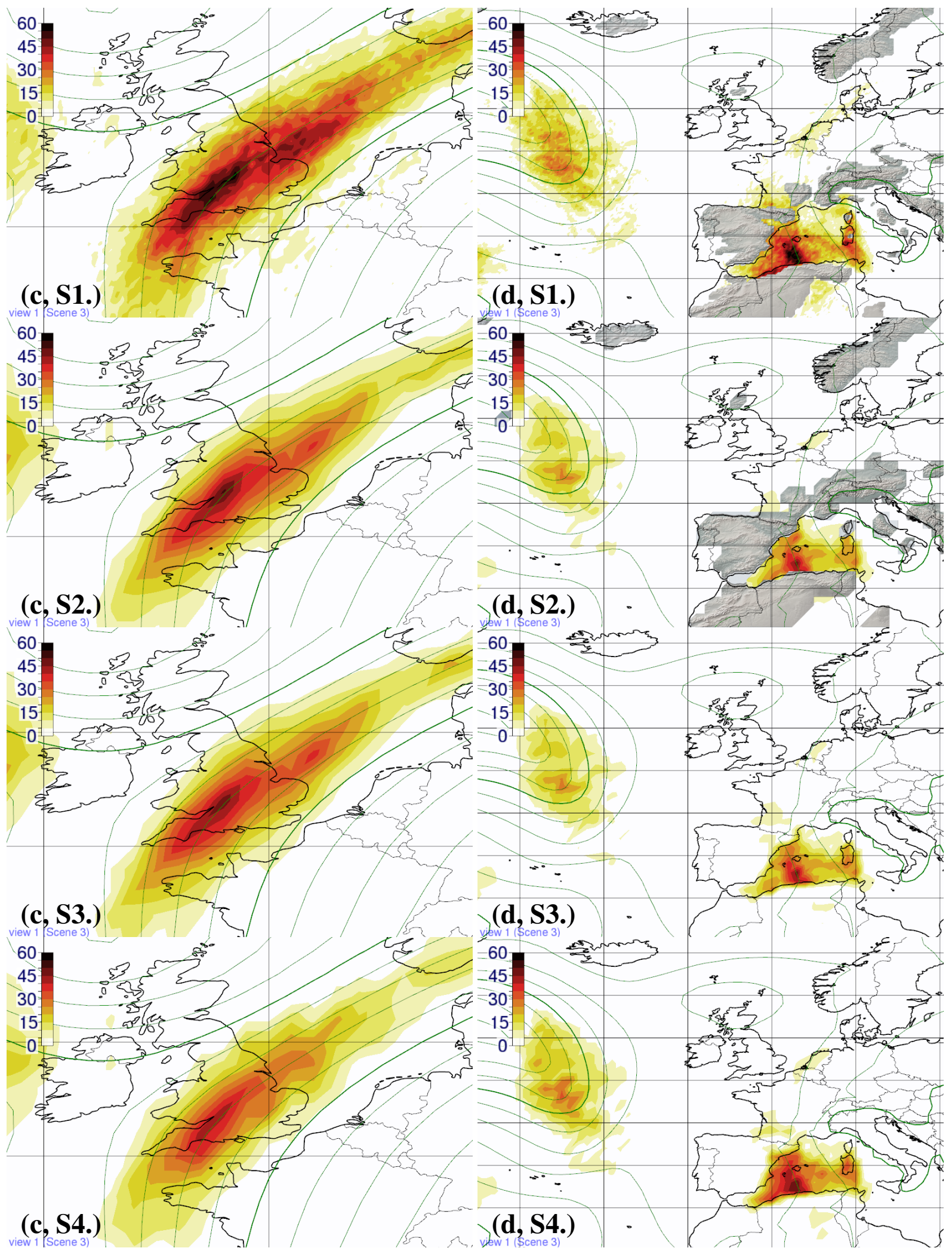

Figure 6. Same as Fig. 5, but showing (c) horizontal section of $p(\mathrm{WCB})$ at $410 \mathrm{hPa}$; (d) horizontal section of $p(\mathrm{WCB})$ at $950 \mathrm{hPa}$; colour coding in \%; green contour lines show ensemble mean geopotential height. 

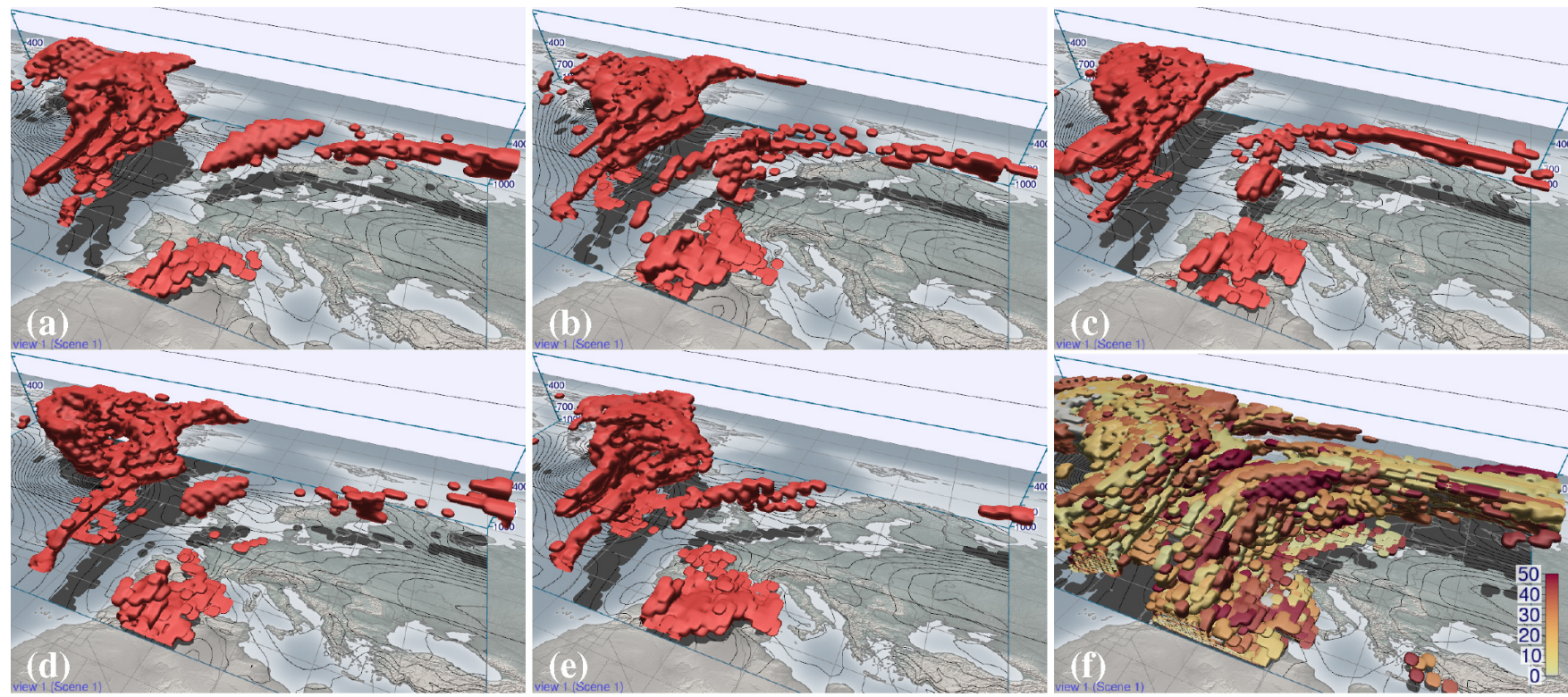

Figure 7. WCB features (binary grids $B^{m}$ ) of further members $m$ of the forecast shown in Fig. 3b. Members (a) 2, (b) 4, (c) 34 , (d) 36 and (e) 42. Note that location and shape of the WCB features vary strongly. (f) WCB features of all 51 members of the ensemble, visualized in a single image and distinguished by colour (colour coding denotes member number). Black contour lines in all images show sea level pressure of the corresponding member (of the ensemble mean in $\mathbf{f}$ ).

causes is very important for making decisions on potential flight routes.

The issue can be approached by looking at the individual ensemble members, as illustrated in Fig. 7. While due to limited print space, Fig. 7 only shows a small selection of members, we indeed find that much more than $30 \%$ of the members predict a WCB feature. However, it is difficult for a human user to remember how many of the 51 members showed a WCB feature. Visualizing the WCB features of all members in a single view (Fig. 7f) results in massive clutter and, thus, does not reveal insight.

We are interested in the following information: given a region bounded by a probability isosurface, how many individual ensemble members predict a WCB feature that overlaps with this region and that, thus, contributes to the probability value at any of the grid points inside the isosurface? To determine this number of members, we propose a method that applies region growing to identify the grid points inside the isosurface, then uses the members' binary grids $B^{m}$ to determine which members have contributed. To efficiently make use of the $B^{m}$, we condense the binary grids into bitfields that are stored together with the probability volume. For the current example and for the 51 members of the ECMWF ensemble, each grid point $p(\mathrm{WCB})_{k j i}$ is augmented by a bitfield stored in a 64 bit integer variable ( 1 bit for each member). The bitfields are generated during evaluation of the probability criterion (in this case, step (3) in Sect. 2).

Figure 8 illustrates the approach. In a hypothetical ensemble of ten members, nine members predict a WCB feature

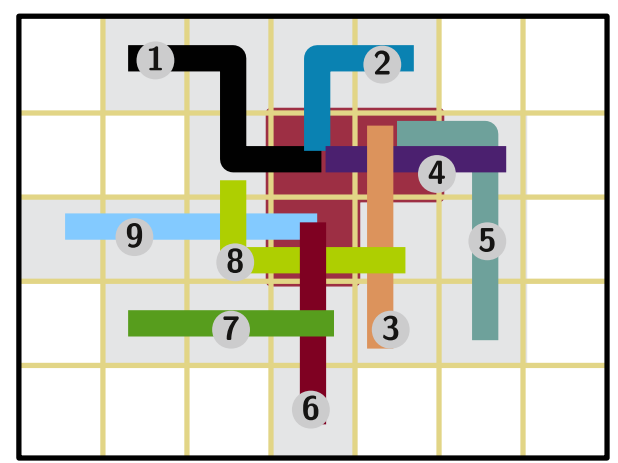

Figure 8. Schematic 2-D example of a case in which many ensemble members predict a WCB feature but spatial variation causes low probability values. Consider an ensemble of ten members, of which nine members predict a WCB feature (depicted by the different coloured and numbered lines). In the example, only a maximum of three features overlap in any grid cell (resulting in a maximum probability of $30 \%$; red grid cells). By storing the indices of all members that contribute to a given grid cell, our method is able to determine the members that contribute to a probability region. In the example, eight members (that is, $80 \%$ ) contribute to the red region. The grey grid cells illustrate the $10 \%$ region.

(coloured bars). However, the maximum probability value that occurs is $30 \%$ (red region). To determine the contribution to the region, the algorithm scans the volume for grid points exceeding the $30 \%$ value. Starting from the first identified point, a region growing algorithm determines all grid points belonging to the red region. Combining the bitfields 
of the identified points with a bitwise "or"-operation reveals that in total, members $1,2,3,4,5,6,8$ and 9 , thus $80 \%$ of the ensemble, contribute to the region. We hence know that much more than $30 \%$ of all members predict a WCB. The information is stored for each of the identified grid points in a separate data field, the "contribution volume". It needs to be recomputed every time the probability isovalue changes. For example, applying the algorithm to the white $10 \%$ region in Fig. 8 yields a contribution of $90 \%$.

The contribution volume can be used in visualizations of $p$ (WCB) to colour a probability isosurface according to the number of members that contribute. Figure 9 shows the application of the method to the WCB forecast from Fig. 3c, set-up (S3). Whenever an isosurface point is identified and visualized (cf. Part 1, Sect. 4.3, for the employed raycasting algorithm), the eight data points that enclose the isosurface position are sampled. Since the isosurface value is interpolated from these eight points, at least the point with the maximum probability value is located inside the isosurface, and the point with the lowest value is located outside the isosurface (otherwise no crossing could be found between the points). Thus, by sampling the contribution volume at the grid point with the maximum value (and exploiting the fact that all grid points of a contiguous structure in the contribution volume carry the same value) the number (or percentage) of contributing members can be obtained. Indeed, Fig. 9c shows that about $85 \%$ of the example's ensemble members contributed to the $30 \%$ isosurface - an immediate hint to the forecaster to have a closer look at the predicted structure.

In addition, region growing can be applied to yield information on how many disjoint WCB features contribute from a particular member, and how the sizes of these features compare to the size of the region bound by the probability isosurface. The diagram in Fig. 9d is displayed by Met.3D when the user selects an isosurface with the mouse pointer. It shows the sizes of the WCB features in the individual members in a stacked box plot. The size of the probability isosurface is displayed by the red line. Single features are divided into solid bars, depicting the fraction of the feature that overlaps with the probability isosurface, and a transparent bar, depicting the full size of the feature. If more than one feature contributes from a given member, each disjoint feature is shown in a different colour. For the example in Fig. 9, this information reveals further insight: first, most members contribute exactly one contiguous feature; second, these features are for the most part substantially larger than the isosurface region (also compare the size of the probability isosurface to the WCB features in Fig. 7). We infer that most members' features indeed represent WCB events. A WCB is hence very likely to occur.

Of course, the method can also be applied to probability fields other that $p(\mathrm{WCB})$; similarly low probabilities can also occur for features derived from other NWP fields.

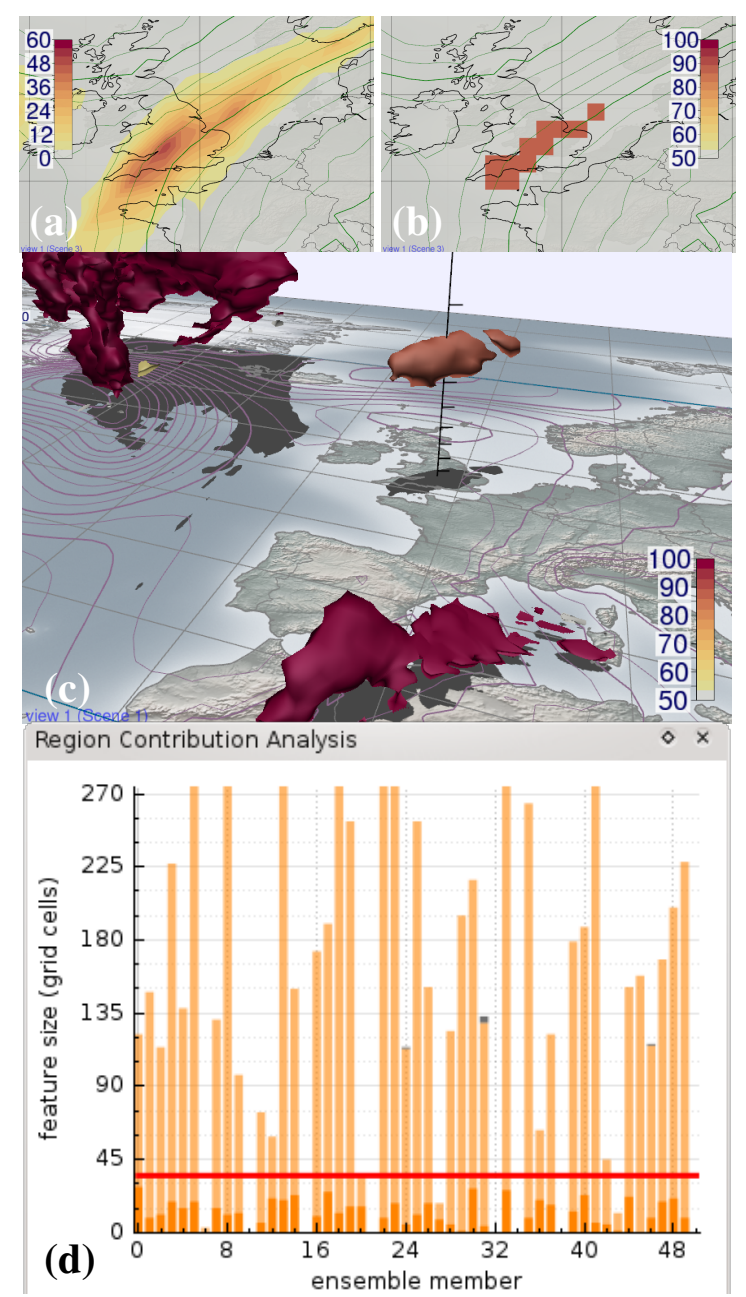

Figure 9. Application of the region contribution algorithm to the WCB forecast from Fig. 3c. (a) Horizontal section of $p(\mathrm{WCB})$ at $415 \mathrm{hPa}$ over southern England (colour coding in \%). (b) Grid boxes that intersect with the $415 \mathrm{hPa}$ surface and that exceed the isosurface threshold of $30 \%$ (the red isosurfaces in Fig. 3c), coloured by the percentage of contributing members as identified by the region growing algorithm (colour coding in \%). Green contour lines in (a) and (b) show ensemble mean geopotential height. (c) The $30 \%$ isosurfaces of Fig. $3 \mathrm{c}$ coloured by the percentage of contributing members. Purple contour lines show ensemble mean sea level pressure. (d) Size (in grid cells) of WCB features in the members contributing to the $30 \%$ isosurface above southern England. If multiple features contribute from a given member, they are stacked using different colours (in the example, small secondary features exist in members 24, 31 and 46). The bar of each feature is divided into total feature size (light colour) and the fraction of the feature that overlaps with the $30 \%$ isosurface (solid colour). The red horizontal line marks the size of the $30 \%$ isosurface.

\section{Case study}

At this point, all visualization and analysis methods are available that are required to use Met.3D to answer the forecast questions listed in the introduction. This section 

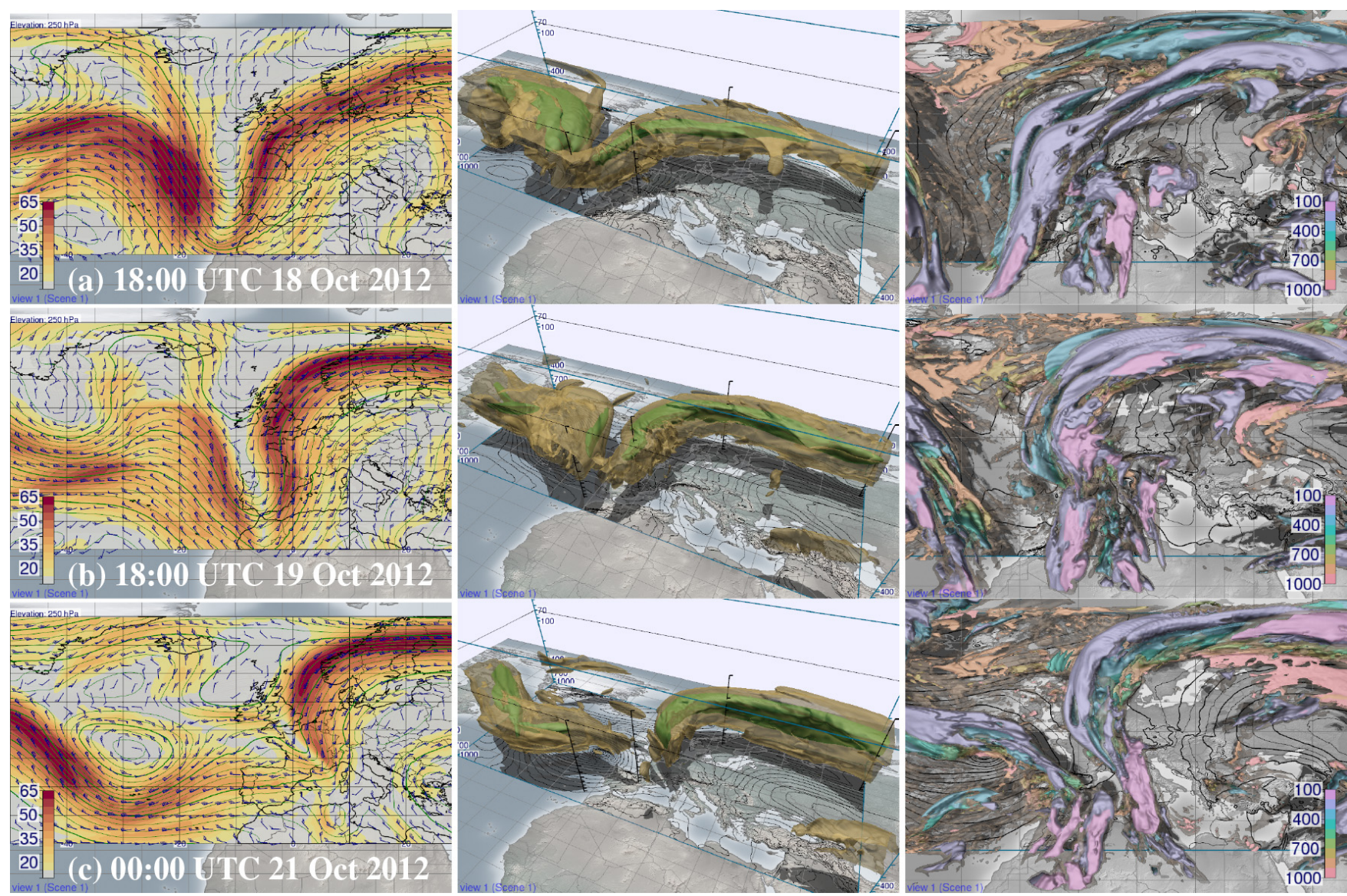

Figure 10. Time sequence of (left) horizontal section with contour lines of geopotential height and filled contours of wind speed (ms $\left.{ }^{-1}\right)$ at $250 \mathrm{hPa}$, (middle) jet stream (opaque isosurface $50 \mathrm{~ms}^{-1}$, transparent isosurface $30 \mathrm{~ms}^{-1}$, and black contour lines of sea level pressure) and (right) clouds (opaque isosurface cloud cover fraction of 0.7 , transparent isosurface cloud cover fraction of 0.2 , and black contour lines of sea level pressure). Colour coding in the right panel denotes cloud elevation in hPa. Deterministic forecast from 00:00 UTC, 15 October 2012, valid at (a) 18:00 UTC, 18 October 2012, (b) 18:00 UTC, 19 October 2012 and (c) 00:00 UTC, 21 October 2012.

demonstrates how Met.3D can be used in practice. The presented case study revisits the TNF forecast case for 19 October 2012, a case that has already been used in the previous sections and in Part 1 and that is also discussed in Schäfler et al. (2014). We supplement the case study with a video accompanying this paper, as it helps convey the full value of Met.3D's interactive 3-D visualizations. The video contains this section's static figures, as well as additional content, in animated form. It is intended to be used side-by-side with the paper. Start times for the video are provided throughout the following text. To compute $p$ (WCB), set-up (S3) from Sect. 3 is used.

Assume the forecast activities to take place on Monday, 15 October 2012. The ensemble and deterministic predictions initialized at 00:00 UTC on that day, as well as the preceding model runs, are available to the forecaster (in the following, we abbreviate forecast valid times as " $12 Z / 19$ " for 12:00 UTC, 19 October 2012, and forecast initialization (or base or run) times as "IT00Z/15" for 00:00 UTC, 15 October
2012). We are interested in areas that favour WCB development in central Europe, being reachable with the Deutsches Zentrum für Luft- und Raumfahrt (DLR) Falcon aircraft from the campaign base in Oberpfaffenhofen, southern Germany. Due to requirements from air traffic authorities, potential flight routes need to be announced at least three days in advance of a flight. Hence, our aim is to explore the atmospheric situation in order to evaluate suitable flight conditions towards the end of the week.

\subsection{Weather situation}

Our first step is to study the large-scale weather situation in the deterministic high-resolution forecast to analyse whether a promising synoptic situation will develop (forecast question A). The upper level flow is of particular interest. WCBs frequently occur on the leading edge (i.e. downstream) of troughs (where low pressure systems develop), and WCB outflow is often associated with jet streaks. We start with a Met.3D configuration featuring three views: a horizon- 
tal section of wind speed and geopotential height (initially placed at jet stream level at $250 \mathrm{hPa}$ ), 3-D isosurfaces of wind speed and 3-D isosurfaces of cloud cover. We explore the time period from Wednesday, 17 October, to Sunday, 21 October. Figure 10 shows screenshots of the individual views at three selected time steps. To capture the 3-D spatial structure of the jet, the isosurfaces of wind speed are visualized at 30 and $50 \mathrm{~ms}^{-1}$. Cloud cover is visualized by isosurfaces at 0.2 and 0.7 , the latter coloured by elevation. Both 3-D views contain contour lines at surface level showing the mean sea level pressure. The video shows the Met.3D window with the full time animation.

A number of events of interest to our objectives can be observed: a distinct trough over the Atlantic moves eastward and narrows over time. At the same time, high pressure over central and eastern Europe intensifies. At upper levels, a pronounced jet stream extends from Spain over southern England to Scandinavia, causing strong winds over western Europe blowing from a southerly direction. On the leading edge of the trough, upper level cirrus clouds are embedded in the jet, whereas upstream, i.e. on the rear side of the trough, only scattered low-level clouds are present. Further upstream (south of Greenland in Fig. 10), the large-scale flow and cloud field are perturbed by the extratropical transition of former Hurricane Rafael (cf. Fig. 1 in Part 1; cloud field visible in the video). It approaches from the south and transforms into an extratropical cyclone. The leading edge of the trough, covering France and southern England, would be well reachable with the Falcon.

Before we explore further forecast data, we obtain information about the uncertainty of the forecast (forecast question B). First, we check the consistency of the deterministic forecast by comparing the currently used forecast (IT00Z/15) to the two previous runs from IT12Z/14 and IT00Z/14. The video (at 00:36 min) shows how the forecast runs are toggled for the forecast valid at 18Z/19. While the IT00Z/15 and IT12Z/14 runs show a fairly consistent situation, the trough is much broader in the IT00Z/14 forecast. Also, the strong jet on its leading edge has a different shape and is located further east and further north. For specifying a flight route, this spatial uncertainty is an important factor.

To get a more comprehensive picture, we explore the ensemble forecast of IT00Z/15 for occurrence, location and intensity of trough and jet in the individual members. Figure 11 shows selected ensemble members and the ensemble mean of the jet stream visualization for the forecast valid at 18Z/19 (the animation over the members is contained in the video at 01:14 min). The jet over Europe is present in all members with similar intensity. However, we observe variation in shape and location that is in part stronger than the difference between the IT00Z/14 and IT00Z/15 deterministic forecasts. Nevertheless, the majority of the members predict a comparable jet structure over Europe. This also becomes apparent in the ensemble mean, which despite averaging features a jet core of over $50 \mathrm{~ms}^{-1}$. In contrast, the variation observed in the jet structure further upstream over the central North Atlantic is larger, indicating that the predicted evolution of the extratropical transition of Hurricane Rafael is very uncertain. Here the $50 \mathrm{~ms}^{-1}$ signal is smoothed out in the mean.

In summary, we conclude that at least parts of the region approximately covering France, southern England and Benelux will be located on the downstream side of the trough.

\subsection{Warm conveyor belt occurrence}

Next, we examine the $p$ (WCB) data to determine whether a suitable WCB event is likely to occur in our region of interest (forecast question C). Figure 12 shows selected time steps from the IT00Z/15 forecast; the corresponding animation is shown in the video at 01:55 min. We choose an initial selection criterion of $\Delta p=500 \mathrm{hPa}$ in $\Delta t=48 \mathrm{~h}$ and visualize the predicted fields with a 3 -D isosurface of a low probability $(10 \%)$. To track the temporal evolution of the $p$ (WCB) field inside the isosurface (in particular the evolution of the maxima), the 3-D normal curves proposed in Part 1, Sect. 3.4, are used $^{2}$.

Indeed, we find that on both 18 and 19 October, WCB air masses are likely to ascend on the leading edge of the trough over France and southern England. These air masses are potentially of interest to a research flight. Since the normal curves reveal larger probabilities on 19 October, we focus on this day. At 12Z/19 (Fig. 12b) and 18Z/19 (Fig. 12c), the ascent signal is most apparent in the prediction. At 00Z/19 and 06Z/19 (Fig. 12a), the air mass is still close to the surface and too far south to be reached by a single Falcon flight. At 00Z/20 (Fig. 12d) and 06Z/20, the air mass has reached upper levels and WCB activity is dominated by outflow. For the campaign objectives, the time around 12Z/19 and 18Z/19 is most interesting to us: the air is ascending and hence meteorologically active (precipitation is associated with the ascending phase of a WCB), and it is located in an area that can be well reached by the Falcon. The 3-D visualization allows one to judge the vertical extent, shape and elevation of the region of high probability. The normal curves, coloured by probability, reveal that the maximum values of $p(\mathrm{WCB})$ on 19 October are of the order of 20 to $30 \%$. By moving the camera and using vertical poles, we see that the region enclosed by the $10 \%$ isosurface is tilted westwards (left column of Fig. 12; video at 02:29 min). At 18Z/19, the maximum is located at around $400 \mathrm{hPa}$.

Due to the low magnitudes of $p(\mathrm{WCB})$, we next intend to clarify (a) whether indeed only a few ensemble mem-

\footnotetext{
${ }^{2}$ Normal curves are well-suited in this case to obtain an overview of the situation, as the magnitudes of maximum $p(\mathrm{WCB})$ values and their variation between time steps are not known beforehand; hence it is difficult to choose a suitable value for an inner opaque isosurface as done for the jet visualization (see Part 1, Sect. 3.4). Normal curves converge at local extrema and hence at a glance highlight maxima, regardless of their magnitude.
} 

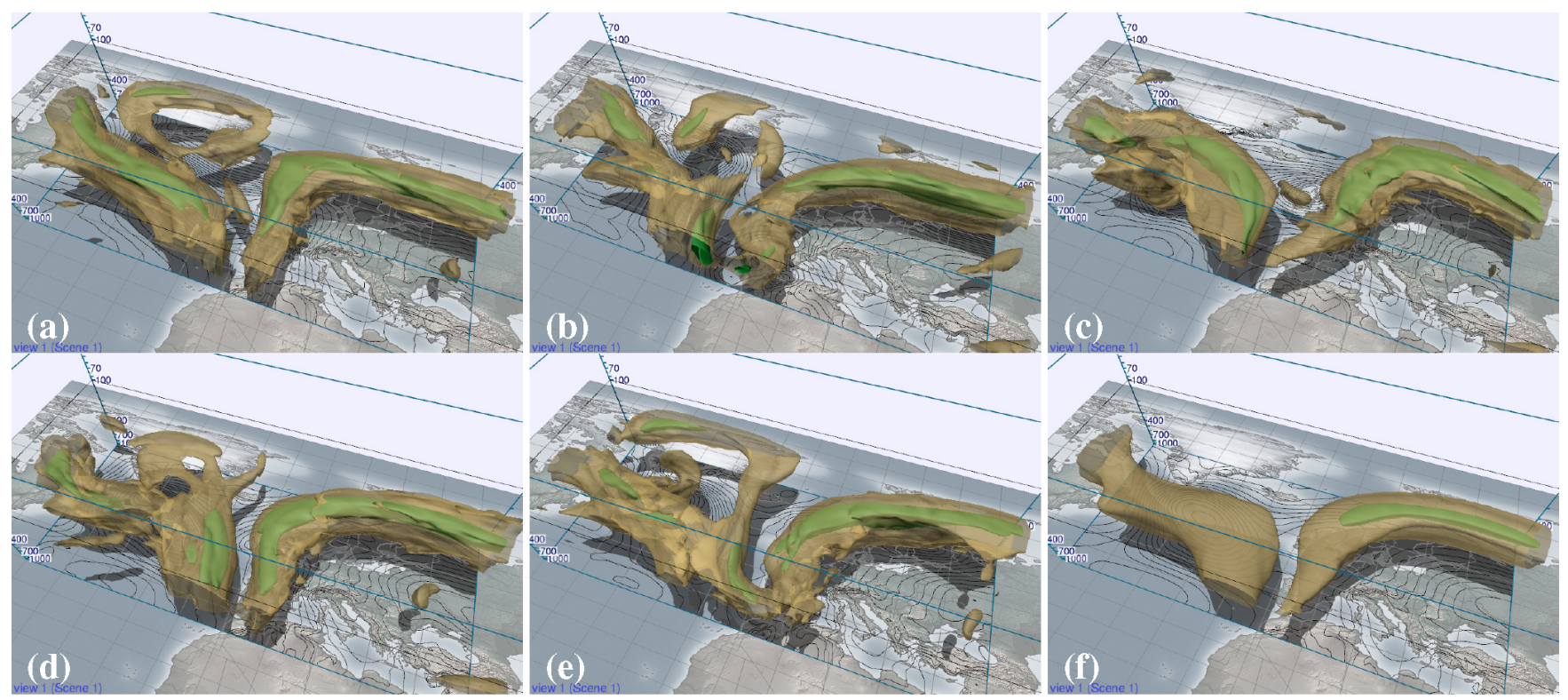

Figure 11. Navigation through the ensemble. Members (a) 27, (b) 33, (c) 37, (d) 43, (e) 45 and (f) the ensemble mean of horizontal wind speed (forecast from 00:00 UTC, 15 October valid at 18:00 UTC, 19 October 2012). Shown are the $50 \mathrm{~ms}^{-1}$ (green opaque) and $30 \mathrm{~ms}^{-1}$ (yellow transparent) isosurfaces. Black contour lines show sea level pressure.

bers predict the WCB, and (b) how the predicted probability changes with a changing selection criterion (forecast question D). Figure 13a shows a screenshot of Met.3D with the region contribution analysis (Sect. 4) applied to the forecast valid at $18 \mathrm{Z} / 19$ (video at $02: 42 \mathrm{~min}$ ). A $20 \%$ isosurface is used to capture the regions of maximum predicted $p(\mathrm{WCB})$. Indeed, for both $12 Z / 19$ (not shown) and 18Z/19 the analysis confirms that over $85 \%$ of the ensemble members have contributed to the $20 \%$ probability region over the English Channel. The difference between 20 and $85 \%$ indicates large spatial variation in the ensemble. Also, the histogram (on the right side of Fig. 13a) shows that the majority of individual WCB features that overlap with the $20 \%$ isosurface cover a larger volume than the resulting probability region itself. This implies that the regions that experience ascent in the individual members are larger than the region enclosed by the isosurface. To validate these findings, we animate over the individual members (Fig. 13b, c, and d; video at 03:16 min). Indeed, almost all members predict a WCB feature on the leading edge of the trough. However, as expected, the variability in shape and location of the predicted features is very large. In addition to members in which the WCB air ascends at $18 \mathrm{Z} / 19$, members in which the air is already in the outflow stage (elongated features at jet stream level) or still in the inflow stage (close to the surface) are equally present. This indicates additional temporal uncertainty. Hence, while there seems to be a good chance to sample WCB air on 19 October in the region covering western France to southern England, the location in space and time of the WCB ascent is still uncertain in the IT00Z/15 forecast.
To judge the strength of the predicted ascent, we modify the trajectory selection criterion. Figure 14 (video at 03:38 $\mathrm{min}$ ) shows how the predicted $p(\mathrm{WCB})$ changes with $\Delta p$. By decreasing $\Delta p$ (Fig. 14a), we can confirm a high likelihood of ascending air masses in the region of interest ${ }^{3}$; the probability increases with decreasing $\Delta p$. Increasing $\Delta p$ (Fig. 14b) reduces the predicted probabilities. However, the location of the maximum remains at the same position. The region in which high probabilities for ascending air masses are forecast is hence also the region in which the strongest updrafts occur.

\subsection{WCB characteristics}

The next goal is to characterize the predicted ascent with respect to related atmospheric processes (forecast question E). We take a closer look at the WCB trajectories of the ensemble control run and visualize the trajectory particle positions at single time steps. Animation over the time steps of the trajectories computed forward and backward from 18Z/19 reveals that the air that at $18 \mathrm{Z} / 19$ has ascended to the region over the Channel originates from the ABL over the western Mediterranean Sea and northwestern Africa around 18Z/18 (Fig. 15a, video at 04:10 min). It is lifted over Spain in the early hours of 19 October and over the course of the day continues its ascent over western France, the Channel and southern England (Fig. 15b, c). By vertically shifting a hori-

\footnotetext{
${ }^{3}$ Note that the normal curves are again advantageous for this interaction as they allow one to visually track the location and magnitude of maximum probabilities despite the changing magnitudes.
} 

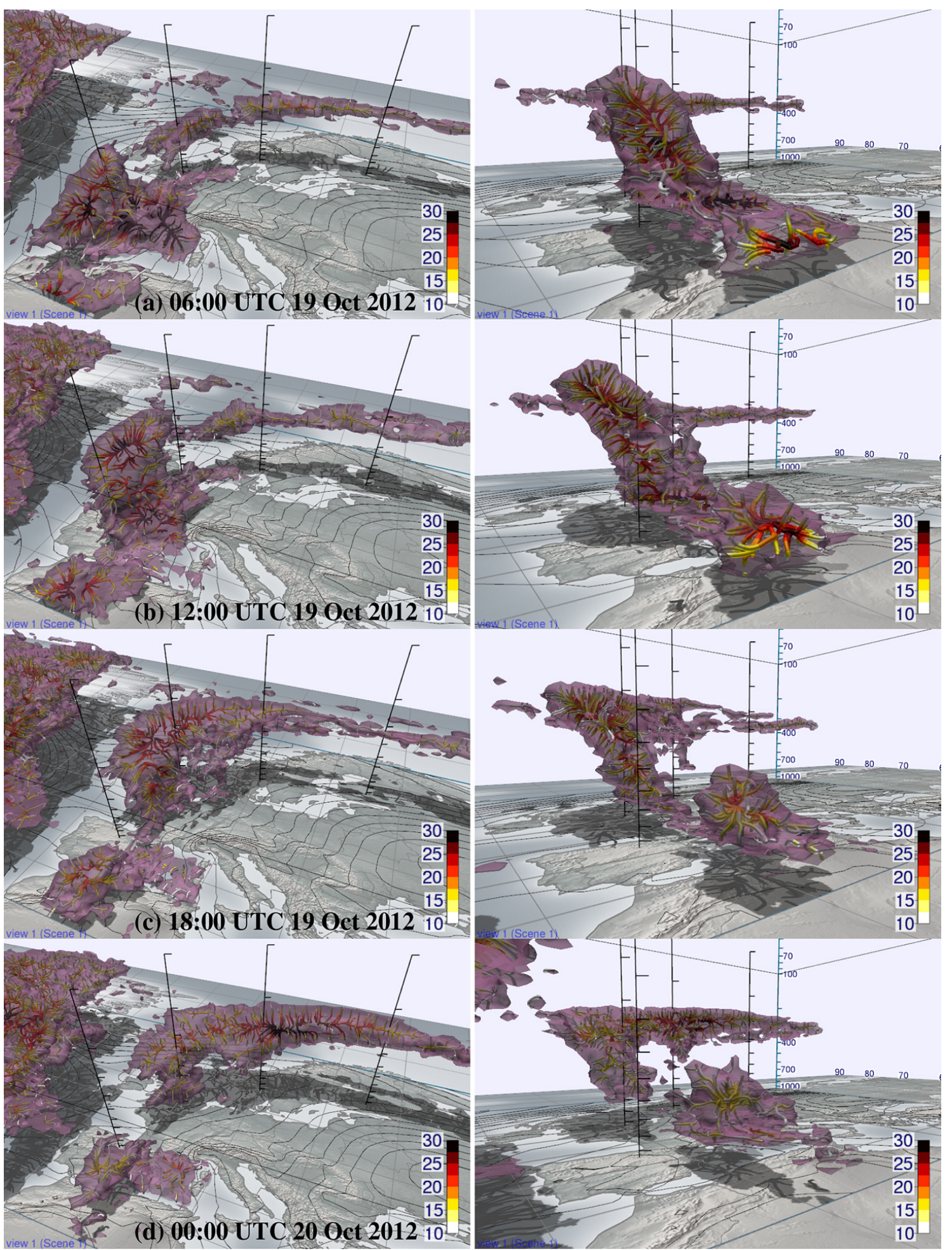

Figure 12. Subsequent time steps of $p$ (WCB) (computed with DF-T set-up (S3)), rendered from different viewpoints. Forecast from 00:00 UTC 15 October 2012, valid at (a) 06:00 UTC, (b) 12:00 UTC, (c) 18:00 UTC, 19 October 2012 and at (d) 00:00 UTC, 20 October 2012. Trajectory filtering is set to $500 \mathrm{hPa}$ in $48 \mathrm{~h}$. The purple transparent isosurfaces show a probability of $10 \%$. The interior structure of the isosurfaces is visualized using the 3-D normal curves approach proposed in Part 1, Sect. 3.4. The normal curves follow the gradient of the $p(\mathrm{WCB})$ field and converge at local maxima. They are coloured by probability (\%) to allow one to visually track probability maxima (red to black convergence zones). Black surface contour lines show ensemble mean sea level pressure. 


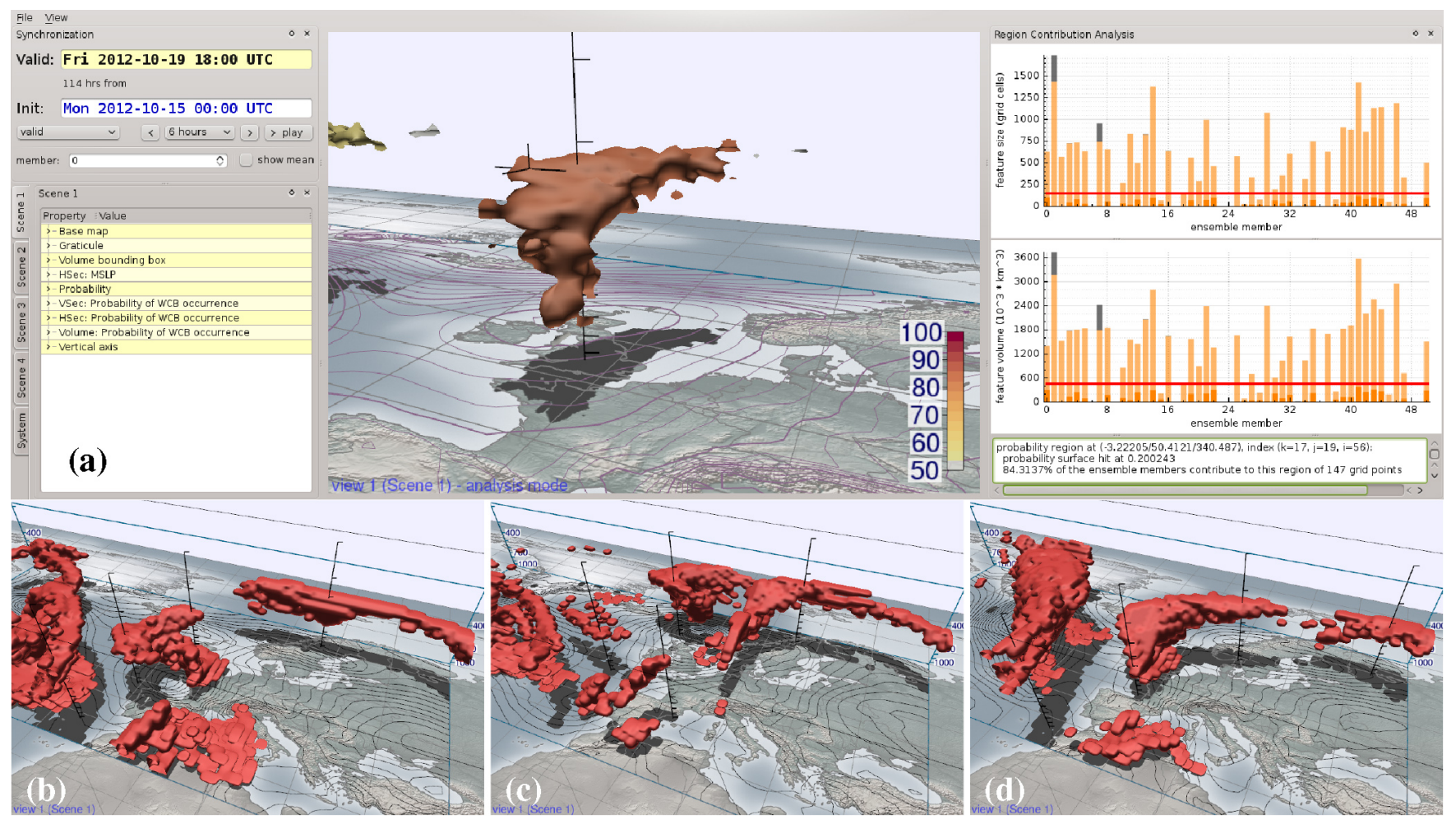

Figure 13. Region contribution analysis applied to the ensemble forecast from 00:00 UTC, 15 October 2012, valid at 18:00 UTC, 19 October 2012. (a) Screenshot of the Met.3D configuration; $20 \%$ isosurfaces of $p$ (WCB) are coloured by the percentage of contributing members. The contribution distribution of the feature over southern England is shown in the histograms on the right side of the window (feature size in (top) grid cells and (bottom) $10^{3} \mathrm{~km}^{3}$; see Fig. 9 for details on the diagram). (b-d) WCB features (binary grids $B$ ) as predicted by the individual ensemble members (b) 2, (c) 9 and (d) 19. Purple surface contours in (a) and black surface contours in (b-d) show sea level pressure.

zontal section of geopotential height and equivalent potential temperature of the deterministic forecast at 18Z/18 (similar to the ensemble control but chosen here for its added detail), we discover a cyclone over the northern British Isles, and a weaker surface low located on the west coast of France (Fig. 15d; video at 04:28 min). South of Spain, warm and moist air (high equivalent potential temperature) is advected northward. This air mass represents the WCB inflow region; it is subsequently lifted by the WCB. In contrast, on the rear side of the trough, colder and drier air masses over the eastern Atlantic are transported southward to Spain. Over the following $24 \mathrm{~h}$, the cyclone over the British Isles remains stationary, the weaker surface low moves towards Norway (Fig. 15e, f; video at 04:52 $\mathrm{min}$ ). Animation over the ensemble members reveals that most other members predict similar ascents originating from the western Mediterranean Sea and northwestern Africa. Figure 16 reproduces the visualization of Fig. 15c for the members shown in Fig. 13b, c, and d. The trajectory particles that represent the WCB air masses are lifted along similar paths. However, the temporal evolution of the WCBs differs in the members. At 18Z/19, the air masses are at different stages of their ascent.
Figure 17 shows vertical sections of potential vorticity (PV) and cloud cover of the deterministic forecast valid at 18Z/19 (animated in the video at 05:19 min). The dynamic tropopause, as indicated by the $2 \mathrm{PVU}$ (potential vorticity unit) surface, folds along the trough (Fig. 17a). On the rear side of the trough, dry stratospheric air is transported downward. On its leading edge, the tropopause is elevated where it transitions into the anticyclonic region over central Europe. Between 700 and $500 \mathrm{hPa}$, increased values of PV indicate regions of diabatic PV production. They coincide with the cold front that can be identified from the strong gradient in equivalent potential temperature (dense contour lines below the clouds in Fig. 17b). The cold front tilts westward with height, matching the tilted structure of the $p(\mathrm{WCB})$ isosurface described in the previous section. Ahead (east) of the front, predicted cloud cover largely coincides with the location of the WCB. Overall, the situation resembles the classic conceptual WCB model (Browning, 1986). The WCB outflow predicted over the North Sea at $00 \mathrm{Z} / 20$ is related to lower PV values aloft. This is consistent with predicted ice water and cloud cover in this region (not shown). 


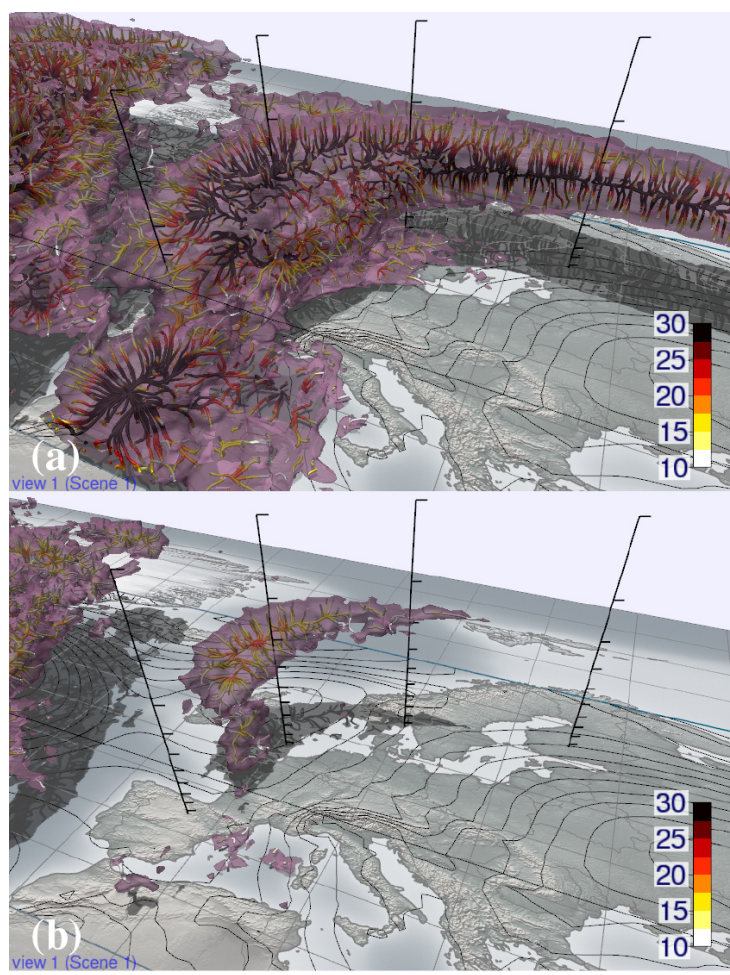

Figure 14. Adjusting the filter criterion for the $p$ (WCB) forecast shown in Fig. 12c (forecast from 00:00 UTC, 15 October 2012, valid at 18:00 UTC, 19 October 2012). Filter criterion of (a) $400 \mathrm{hPa}$ and (b) $550 \mathrm{hPa}$ in $48 \mathrm{~h}$ (in Fig. 12c a criterion of $500 \mathrm{hPa}$ in $48 \mathrm{~h}$ is used). The purple transparent isosurfaces show a probability of $10 \%$. Normal curves inside the isosurfaces are coloured by probability (\%). Black contour lines show ensemble mean sea level pressure.

\subsection{Potential flight segments}

Given the findings from the previous subsections, we interpret the $p(\mathrm{WCB})$ maximum as the most likely location for the predicted WCB event and draft potential flight segments. Figure 18 shows the corresponding Met.3D configuration. For $12 Z / 19$ and $18 Z / 19$, we slide a horizontal section trough the $p(\mathrm{WCB})$ volume to determine precise locations of the maxima (video at 05:42 min). At 12Z/19, maximum probabilities are located above the Pyrenees at low levels, in the Bordeaux area between 700 and $600 \mathrm{hPa}$, and south of Brittany around $400 \mathrm{hPa} ; 6 \mathrm{~h}$ later, the maximum is most prominent above southern England at altitudes around $400 \mathrm{hPa}$. A vertical section is used to explore potential flight segments. It allows one to estimate at which elevation a flight should take place, and, by moving the section, to quickly assess how spatially relocating the leg will impact the expected measurements. In the given case, the 2-D sections suggest flight legs at 12Z/19 over France at elevations between 800 and $600 \mathrm{hPa}$
(WCB ascent) and at 18Z/19 over southern England at elevations around $400 \mathrm{hPa}$ (WCB outflow) ${ }^{4}$.

However, given the uncertainty in the temporal evolution of the WCB (previous section), we need to carefully monitor developments in subsequent forecast runs. Figure 19 (video at $07: 38 \mathrm{~min}$ ) shows the predictions for $18 \mathrm{Z} / 19$ for forecast runs subsequent to the IT00Z/15 run. Over the next 2 days, the ensemble predictions converge toward higher $p(\mathrm{WCB})$ over the English Channel and southern England. The elevation of the predicted maximum in $p(\mathrm{WCB})$ remains approximately constant. Indeed, the research flights conducted during TNF showed that the targeted WCB occurred as predicted (Schäfler et al., 2014).

\section{Conclusions}

Motivated by the forecast requirements of the T-NAWDEXFalcon 2012 campaign, we have demonstrated the feasibility of applying interactive 3-D ensemble visualization to forecasting warm conveyor belt situations during aircraft-based field campaigns. The article extends our work presented in Part 1, in which we have introduced the new open-source 3-D ensemble visualization tool Met.3D. In the present paper, we have proposed methods to compute and to visually analyse 3-D probabilities of WCB occurrence. The techniques have been integrated into Met.3D and are part of the released version 1.0 (see Part 1, Sect. 6, for information on code availability). A case study, revisiting a forecast case that occurred during T-NAWDEX-Falcon, has demonstrated how the methods introduced in the two papers can be used for practical forecasting.

Following the literature, our methods detect WCBs by means of Lagrangian particle trajectories. By computing trajectories for each member of the ECMWF ensemble forecast, a distribution of WCB features is obtained from which probabilities of occurrence can be derived. We have discussed different approaches to trajectory seeding and gridding, and have shown that probabilities derived from trajectories computed at a horizontal grid spacing of $1^{\circ}$ in latitude and longitude capture the same WCB structures as trajectories computed at a higher grid spacing of $0.25^{\circ}$. A proposed visual analysis method supports the interpretation of the probability fields. The method facilitates fast visual estimation of the number of ensemble members that forecast a WCB feature in a region of interest bounded by a probability isosurface. In particular for situations in which the magnitude of observed probabilities is low, the method helps to distinguish the case

\footnotetext{
${ }^{4}$ During TNF, we did not have the vertical $p(\mathrm{WCB})$ information available. We placed the flight along a horizontally pre-defined flight leg over France which appeared to fit well with the 2-D $p$ (WCB) product. We were only able to guess at which altitudes we should fly. In fact, from the 3-D $p$ (WCB) data we find that the flight should rather have been planned south of the pre-defined flight leg.
} 

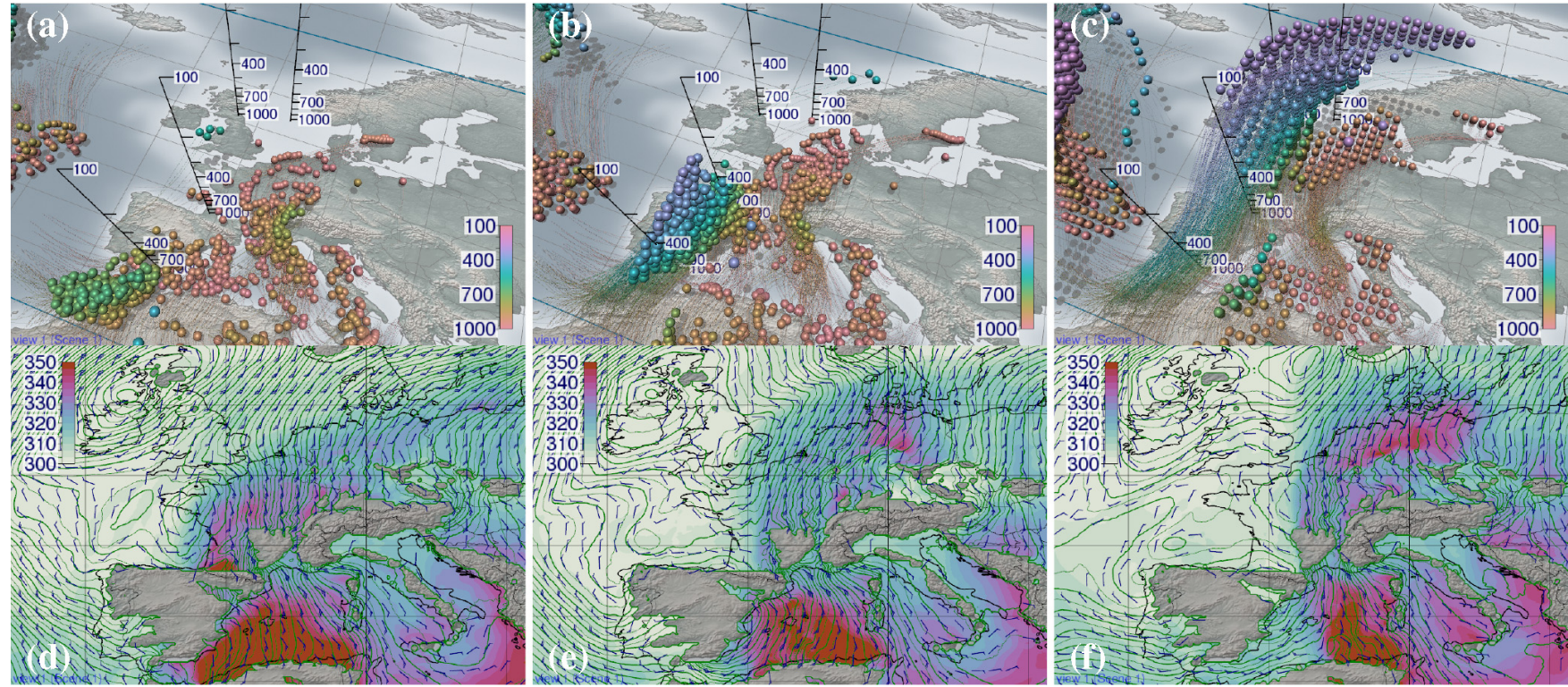

Figure 15. (a-c) Particle positions of the (backward) WCB trajectories of the ensemble control forecast, started at 18:00 UTC, 19 October 2012 and computed on the forecast initialized at 00:00 UTC, 15 October 2012. Colour codes pressure elevation in hPa. (d-f) Horizontal sections of geopotential height (contour lines), wind barbs and equivalent potential temperature (colour coded in K) of the deterministic forecast from 00:00 UTC, 15 October 2012 at $950 \mathrm{hPa}$. Forecasts are valid at (a, d) 18:00 UTC 18 October 2012, (b, e) 06:00 UTC, 19 October 2012 and (c, f) 18:00 UTC, 19 October 2012.
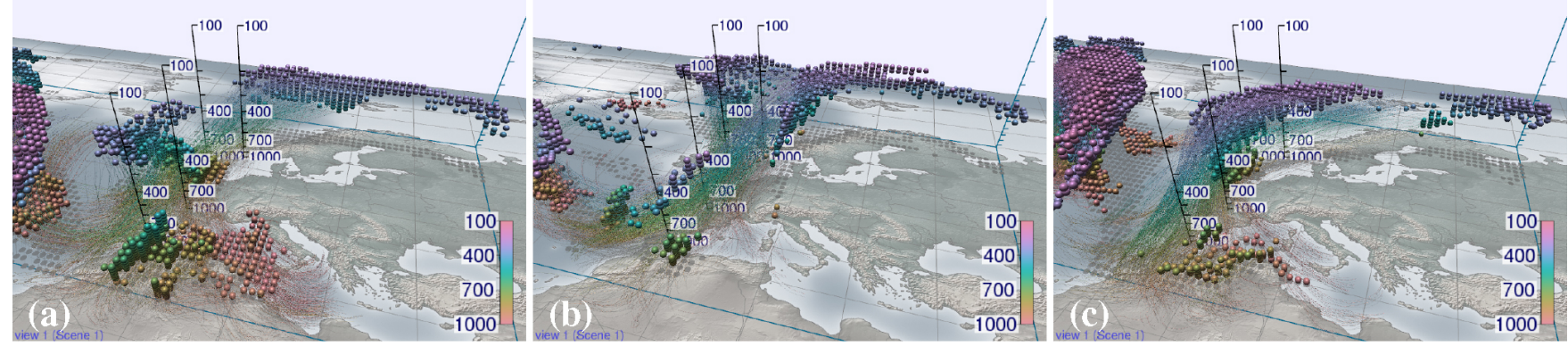

Figure 16. The same as Fig. 15c, but for the ensemble members (a) 2, (b) 9 and (c) 19. Also compare to the visualizations of the corresponding binary grids $B$ shown in Fig. 13b-d.

in which only few members predict a WCB but at approximately the same location, from the case in which many members predict a WCB but the spatial variation is high. The method can be applied to probabilities of other features as well.

With Met.3D and the proposed WCB methods, we are now able to analyse ensemble prediction data in a way previously impossible. Three of us (M. Rautenhaus, C. M. Grams, A. Schäfler) have been actively involved in forecasting during aircraft-based field campaigns. With respect to the case study and our experience in research flight planning, we note a few conclusions from our work, reflecting the authors' opinions.

1. Combination of 2-D and 3-D visualization methods gives a more complete picture of the forecast atmo- sphere; 3-D elements can depict different aspects of the data than horizontal and vertical 2-D sections alone. For example, usage of isosurfaces and normal curves allows for very fast initial judgement of the predicted WCB situation. However, we would not want to abandon the familiar 2-D sections; for many tasks (obtaining quantitative information, visualizing multiple forecast parameters in the same plot, analysing the vertical structure of the atmosphere along a flight segment) they are superior to 3-D methods. If 3-D visualization is used, achieving good spatial perception is important, as we have discussed in Part 1.

Furthermore, while we think that 3-D visualization helps to understand the atmospheric situation in many cases, it does not work equally well for all forecast vari- 

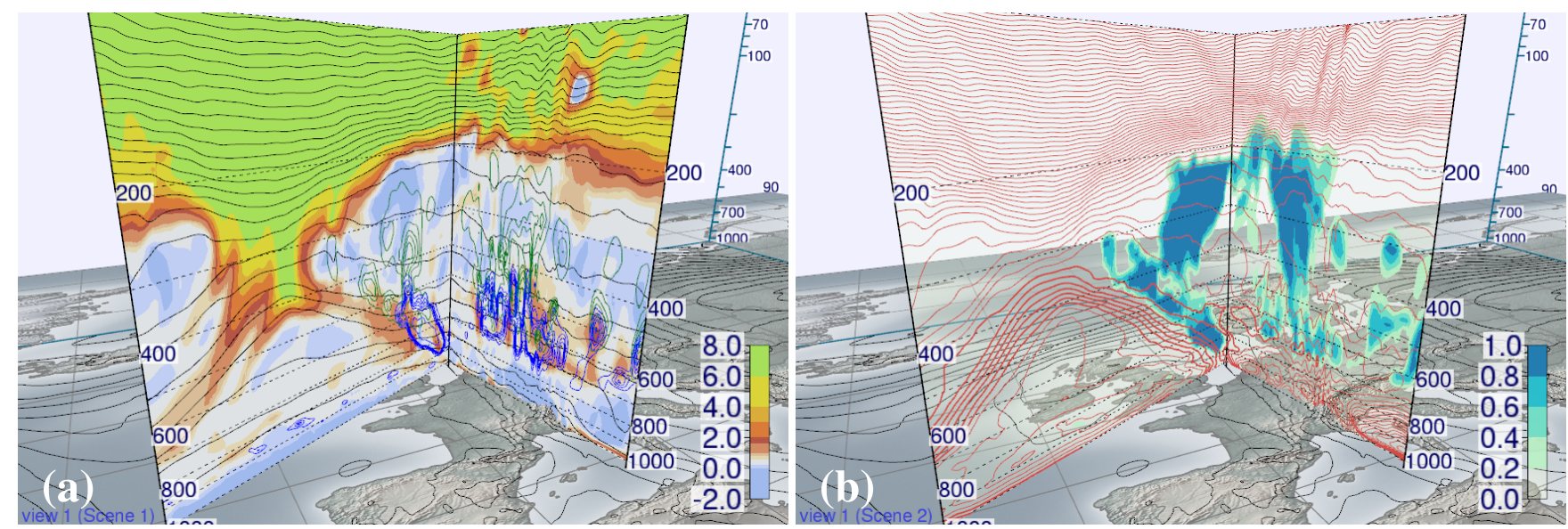

Figure 17. (a) Vertical section of potential vorticity (colour coding in PVU; red colours in the left plot mark the 2 PVU surface and thus the dynamic tropopause), potential temperature (grey contour lines), liquid and ice water content (blue and green contour lines). (b) Vertical section of cloud cover fraction (colour coding) and equivalent potential temperature (red contour lines). Black surface contour lines in both (a) and (b) show sea level pressure. Deterministic forecast from 00:00 UTC, 15 October 2012, valid at 18:00 UTC, 19 October 2012.

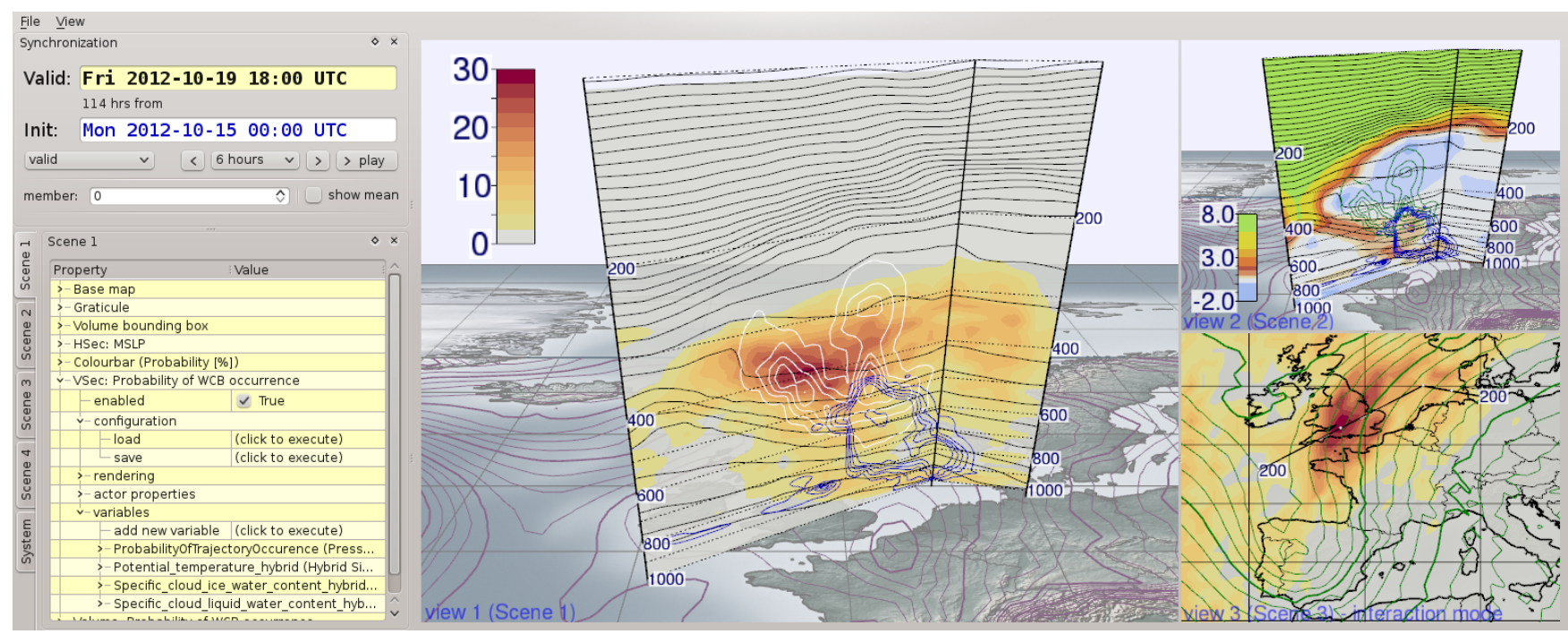

Figure 18. Planning potential flight legs with Met.3D (ensemble forecast from 00:00 UTC, 15 October 2012, valid at 18:00 UTC, 19 October 2012). The large view in the middle shows a vertical section of $p$ (WCB) (colour scale in \%), potential temperature (black contour lines), liquid and ice water content (blue and white contour lines). Purple surface contours show ensemble mean sea level pressure. The small view on the upper right shows a vertical section of potential vorticity (same colour coding and contour lines as in Fig. 17a). The small view on the lower right shows a horizontal section at $390 \mathrm{hPa}$, showing $p$ (WCB) (same colour scale as in the large view) and contour lines of ensemble mean geopotential height. The maximum $p(\mathrm{WCB})$ along the proposed leg can be found over southern England at around $400 \mathrm{hPa}$. The vertical section of PV shows how a flight at that altitude, going westward, would penetrate the tropopause shortly after sampling the WCB.

ables. For the isosurfaces of wind speed and WCB probability used in the case study, 3-D visualization is wellsuited. For variables that highly fluctuate in space (as is often the case for variables depending on moisture, such as relative humidity), isosurfaces are problematic. For these cases, additional methods that help the user focus on the regions and features of interest will need to be developed.
2. One of the primary advantages of Met.3D is the high pace at which a forecast can be explored. Interactivity, the possibility for the user to change a parameter that affects the visualization and to receive immediate visual feedback, is key to this property. It facilitates the very fast analysis of static scenes (moving the camera to explore spatial structure of a feature, moving a vertical axis), of dynamic processes (animation over time), 


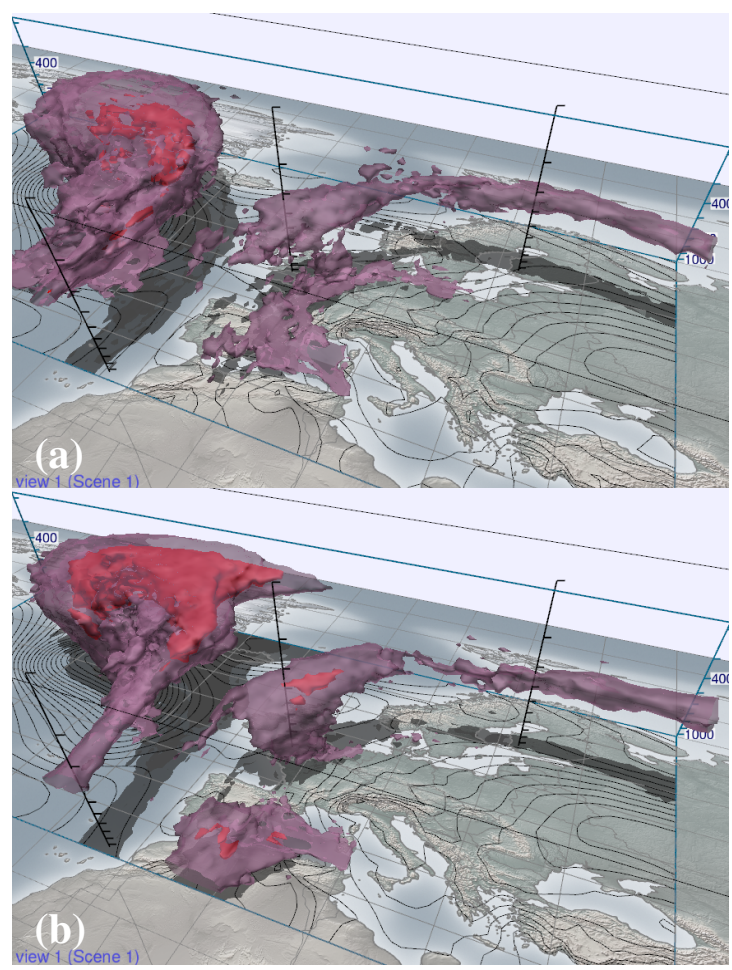

Figure 19. Convergence of $p(\mathrm{WCB})$ with decreasing forecast lead time. Forecasts from (a) 12:00 UTC, 15 October 2012 and (b) 12:00 UTC, 16 October 2012, valid at 18:00 UTC, 19 October 2012. Filter criterion is $500 \mathrm{hPa}$ in $48 \mathrm{~h}$. Isosurfaces show $30 \%$ (red opaque isosurface) and $10 \%$ (purple transparent isosurface). Black surface contours show ensemble mean sea level pressure. The forecast from 00:00 UTC, 17 October 2012 is shown in Fig. 3c.

of uncertainty (animation over the ensemble, comparison of different forecast base times) and of sensitivity (changing a parameter that affects a displayed statistical quantity).

However, we find that while interactivity enables the user to quickly visualize a large amount of data, the user is also confronted with many more images than they would be if they were restricted to, for example, a limited number of horizontal sections. Here, as Trafton and Hoffman (2007) suggest, a virtual "sketchpad" that captures elements discovered by the forecaster and that allows him/her to represent his/her "mental model" of the atmosphere would be useful. The sketchpad could also be used to communicate the findings to colleagues, a common challenge during campaigns.

3. Our methodology to predict probabilities of WCB occurrence illustrates challenges of feature-based approaches to analyse ensemble data. Our region contribution approach helps to interpret the derived probabilities; however, further work will be useful. For example, we would like to automatically obtain information about how features in different members correspond to each other: do other members predict the same situation but shifted in space or in time? Such information would allow one to identify different scenarios forecast by the ensemble, and uncertainty could be differentiated with respect to space and time. Detection and visualization of further 3-D cyclonic features would also be very useful. For a single member we could see at a glance, for example, which WCB transports which air mass along which route, driven by which cyclone and in relation to which front and jet stream. How to meaningfully visualize such features for an entire ensemble to depict their uncertainty is an open research question.

4. A drawback of the Met.3D visualization approach is that since it uses the complete ensemble data set, interactive usage requires the forecast data to be available on the local hard drive. For field campaigns based at remote locations, this is not feasible. In these cases, web based approaches such as DLR's Mission Support System (Rautenhaus et al., 2012) might be the better choice. Alternatively, dedicated ensemble compression schemes might enable more efficient remote handling, or remote visualization solutions such as VirtualGL ${ }^{5}$ could be used to locate data and visualization system at the same site while allowing users to explore the data remotely using a modest internet connection.

We will actively use and further evaluate our developments during upcoming field campaigns, including a future NAWDEX campaign scheduled for 2016. It will again target WCBs. We also intend to continue our work on trajectorybased ensemble analysis. For example, trajectories can be applied to detect further Lagrangian features. Different selection criteria can reveal air masses that have undergone specific physical or chemical processes, or that originate in specific geographic regions. Also, ensemble trajectories can be used to track, for instance, the dispersion of pollutants or volcanic ash. Modified versions of our proposed methods can be used to derive probabilities that reveal forecast uncertainty in regions in which a pollutant or volcanic ash concentrations exceed a critical threshold. In this respect, more complex selection algorithms and the visualization of combined probabilities of multiple features will be challenging.

Considering the ever increasing data volume generated by ensemble weather prediction systems, effective and intuitive visualization methods are and will be important to weather forecasting. The atmosphere is $3-\mathrm{D}$, and while we need to conduct user studies to formally prove the added value through 3-D visualization, in our opinion forecast analysis can be made much more intuitive by using interactive 3-D methods, thus decreasing the time a meteorologist needs to analyse a forecast data set.

\footnotetext{
${ }^{5}$ http://www.virtualgl.org
} 


\section{The Supplement related to this article is available online at doi:10.5194/gmd-8-2355-2015-supplement.}

Acknowledgements. Access to ECMWF prediction data has been kindly provided in the context of the ECMWF special project "Support Tool for HALO Missions". This work was supported by the European Union under the ERC Advanced Grant 291372 SaferVis - Uncertainty Visualization for Reliable Data Discovery. M. Rautenhaus was supported by a grant from Ev. Studienwerk Villigst e.V. C. M. Grams and A. Schäfler were supported by the German Research Foundation (DFG) as part of the research unit PANDOWAE (FOR896).

This work was supported by the German Research Foundation (DFG) and the Technische Universität München within the funding programme

Open Access Publishing.

Edited by: H. Tost

\section{References}

Blakeslee, R., Hall, J., Goodman, M., Parker, P., Freudinger, L., and He, M.: The Real Time Mission Monitor - A Situational Awareness Tool For Managing Experiment Assets, in: NASA Science and Technology Conference 2007, 19-21 June 2007, College Park, MD, 2007.

Browning, K. A.: Radar measurements of air motion near fronts, Weather, 26, 320-340, 1971.

Browning, K. A.: Conceptual models of precipitation systems, Weather Forecast., 1, 23-41, 1986.

Browning, K. A.: Organization of clouds and precipitation in extratropical cyclones, in: Extratropical Cyclones: The Erik Palmén Memorial Volume, edited by Newton, C. W. and Holopainen, E. O., 129-153, Amer. Meteor. Soc., 1990.

Browning, K. A. and Roberts, N. M.: Structure of a frontal cyclone, Q. J. Roy. Meteor. Soc., 120, 1535-1557, 1994.

Buizza, R., Bidlot, J.-R., Wedi, N., Fuentes, M., Hamrud, M., Holt, G., Palmer, T., and Vitart, F.: The ECMWF Variable Resolution Ensemble Prediction System VAREPS, ECMWF Newsletter, 108, 14-20, 2006.

Carlson, T. N.: Airflow through midlatitude cyclones and the comma cloud pattern, Mon. Weather Rev., 108, 1498-1509, 1980.

Ducrocq, V., Braud, I., Davolio, S., Ferretti, R., Flamant, C., Jansa, A., Kalthoff, N., Richard, E., Taupier-Letage, I., Ayral, P.-A., Belamari, S., Berne, A., Borga, M., Boudevillain, B., Bock, O., Boichard, J.-L., Bouin, M.-N., Bousquet, O., Bouvier, C., Chiggiato, J., Cimini, D., Corsmeier, U., Coppola, L., Cocquerez, P., Defer, E., Delanoë, J., Di Girolamo, P., Doerenbecher, A., Drobinski, P., Dufournet, Y., Fourrié, N., Gourley, J. J., Labatut, L., Lambert, D., Le Coz, J., Marzano, F. S., Molinié, G., Montani, A., Nord, G., Nuret, M., Ramage, K., Rison, W., Roussot, O., Said, F., Schwarzenboeck, A., Testor, P., Van Baelen, J., Vincendon, B., Aran, M., and Tamayo, J.: HyMeX-SOP1: The Field
Campaign Dedicated to Heavy Precipitation and Flash Flooding in the Northwestern Mediterranean, Bull. Amer. Meteor. Soc., 95, 1083-1100, 2014.

Dyer, J. and Amburn, P.: Desktop Visualization of Meteorological Data Using Paraview, Kitware Source, 14, 7-10, 2010.

Eckhardt, S., Stohl, A., Wernli, H., James, P., Forster, C., and Spichtinger, N.: A 15-year climatology of warm conveyor belts, J. Climate, 17, 218-237, 2004.

Elsberry, R. L. and Harr, P. A.: Tropical cyclone structure (TCS08) field experiment science basis, observational platforms, and strategy, Asia-Pacific Journal of Atmospheric Sciences, 44, 209-231, 2008.

Flatøy, F., Hov, Ø., and Schlager, H.: Chemical forecasts used for measurement flight planning during POLINAT 2, Geophys. Res. Lett., 27, 951-958, 2000.

Gneiting, T. and Raftery, A. E.: Weather forecasting with ensemble methods, Science, 310, 248-249, 2005.

Grams, C. M., Wernli, H., Böttcher, M., Čampa, J., Corsmeier, U., Jones, S. C., Keller, J. H., Lenz, C.-J., and Wiegand, L.: The key role of diabatic processes in modifying the upper-tropospheric wave guide: a North Atlantic case-study, Q. J. Roy. Meteor. Soc., 137, 2174-2193, 2011.

Grotjahn, R. and Chervin, R. M.: Animated Graphics in Meteorological Research and Presentations, Bull. Amer. Meteor. Soc., 65 , 1201-1208, 1984.

Harrold, T. W.: Mechanisms influencing the distribution of precipitation within baroclinic disturbances, Q. J. Roy. Meteor. Soc., 99, 232-251, 1973.

He, M., Goodman, H. M., Blakeslee, R., and Hall, J. M.: The Waypoint Planning Tool: Real Time Flight Planning for Airborne Science, in: American Geophysical Union 2010 Fall Meeting, \#IN31A-1279, San Francisco, CA, 13-17 December 2010.

Heizenrieder, D. and Haucke, S.: Das meteorologische Visualisierungs- und Produktionssystem NinJo, Promet, 35, 57-69, 2009.

Hibbard, W. L.: Computer-Generated Imagery for 4-D Meteorological Data, Bull. Amer. Meteor. Soc., 67, 1362-1369, 1986.

Hibbard, W. L.: Vis5D, Cave5D, and VisAD, in: The Visualization Handbook, edited byL Hansen, C. D. and Johnson, C., Chapt. 34, 673-688, Academic Press, 2005.

Hibbard, W. L., Santek, D., Uccellini, L., and Brill, K.: Application of the 4-D McIDAS to a Model Diagnostic Study of the Presidents' Day Cyclone, Bull. Amer. Meteor. Soc., 70, 1394-1403, 1989.

Koppert, H. J., Schröder, F., Hergenröther, E., Lux, M., and Trembilski, A.: 3D visualisation in daily operation at the DWD, in: Proceedings of the 6th ECMWF Workshop on Meteorological Operational Systems, 17-21 November 1997, Reading, England, 101-125, 1998.

Kuo, Y.-H., Reed, R. J., and Low-Nam, S.: Thermal Structure and Airflow in a Model Simulation of an Occluded Marine Cyclone, Mon. Weather Rev., 120, 2280-2297, 1992.

Leutbecher, M. and Palmer, T.: Ensemble forecasting, J. Comput. Phys., 227, 3515-3539, 2008.

Madonna, E., Wernli, H., Joos, H., and Martius, O.: Warm Conveyor Belts in the ERA-Interim Dataset (1979-2010). Part I: Climatology and potential vorticity evolution, J. Climate, 27, 3-26, 2014. 
Mass, C. F. and Schultz, D. M.: The Structure and Evolution of a Simulated Midlatitude Cyclone over Land, Mon. Weather Rev., 121, 889-917, 1993.

McCaslin, P. T., McDonald, P. A., and Szoke, E. J.: 3D visualization development at NOAA forecast systems laboratory, ACM SIGGRAPH Comput. Graph., 34, 41-44, 2000.

Murray, D. and McWhirter, J.: Evolving IDV - creating better tools for the community, in: 23th Conference on International Interactive Information and Processing Systems (IIPS) for Meteorology, Oceanography, and Hydrology, 15-18 January 2007, San Antonio, TX, American Meteorological Society, 3B.5, 2007.

Norton, A. and Clyne, J.: The VAPOR Visualization Application, in: High Performance Visualization, edited by: Bethel, E. W., Childs, H., and Hansen, C., Chapt. 20, CRC Press, 415-428, 2012

Pfahl, S., Madonna, E., Boettcher, M., Joos, H., and Wernli, H.: Warm conveyor belts in the ERA-interim dataset (1979-2010). Part II: Moisture origin and relevance for precipitation, J. Climate, 27, 27-40, 2014.

Pomroy, H. R. and Thorpe, A. J.: The evolution and dynamical role of reduced upper-tropospheric potential vorticity in intensive observing period one of FASTEX, Mon. Weather Rev., 128, $1817-$ 1834, 2000.

Rautenhaus, M., Bauer, G., and Dörnbrack, A.: A web service based tool to plan atmospheric research flights, Geosci. Model Dev., 5, 55-71, doi:10.5194/gmd-5-55-2012, 2012.

Rautenhaus, M., Kern, M., Schäfler, A., and Westermann, R.: Threedimensional visualization of ensemble weather forecasts - Part 1 : The visualization tool Met.3D (version 1.0), Geosci. Model Dev., 8, 2329-2353, doi:10.5194/gmd-8-2329-2015, 2015.

Reed, R. J., Kuo, Y.-H., and Low-Nam, S.: An Adiabatic Simulation of the ERICA IOP 4 Storm: An Example of Quasi-Ideal Frontal Cyclone Development, Mon. Weather Rev., 122, 26882708, 1994.

Russell, I., Siemen, S., Ii, F., Kertész, S., Lamy-Thépaut, S., and Karhila, V.: Metview 4 - ECMWF's latest generation meteorological workstation, ECMWF Newslett., 126, 23-27, 2010.

Schäfler, A., Dörnbrack, A., Wernli, H., Kiemle, C., and Pfahl, S.: Airborne lidar observations in the inflow region of a warm conveyor belt, Q. J. Roy. Meteor. Soc., 137, 1257-1272, 2011.

Schäfler, A., Boettcher, M., Grams, C. M., Rautenhaus, M., Sodemann, H., and Wernli, H.: Planning aircraft measurements within a warm conveyor belt, Weather, 69, 161-166, 2014.

Schultz, D. M. and Mass, C. F.: The Occlusion Process in a Midlatitude Cyclone over Land, Mon. Weather Rev., 121, 918-940, 1993.

Spichtinger, P., Gierens, K., and Wernli, H.: A case study on the formation and evolution of ice supersaturation in the vicinity of a warm conveyor belt's outflow region, Atmos. Chem. Phys., 5, 973-987, doi:10.5194/acp-5-973-2005, 2005.

Sprenger, M. and Wernli, H.: The Lagrangian analysis tool LAGRANTO - version 2.0, Geosci. Model Dev. Discuss., 8, $1893-$ 1943, doi:10.5194/gmdd-8-1893-2015, 2015.

Stohl, A.: A 1-year Lagrangian "climatology" of airstreams in the Northern Hemisphere troposphere and lowermost stratosphere, J. Geophys. Res., 106, 7263-7279, 2001.
Trafton, J. G. and Hoffman, R. R.: Computer-aided visualization in meteorology, in: Expertise Out of Context: Proceedings of the Sixth International Conference on Naturalistic Decision Making, edited by: Hoffman, R. R., Chapt. 15, Psychology Press, 337357, 2007.

Treinish, L. A. and Rothfusz, L. P.: Three-dimensional visualization for support of operational forecasting at the 1996 Centennial Olympic Games, in: Proceedings of the 13th IIPS Conference, 2-7 February 1997, Long Beach, CA, 2-8, 1997.

Untch, A. and Hortal, M.: A finite-element scheme for the vertical discretization of the semi-Lagrangian version of the ECMWF forecast model, Q. J. Roy. Meteor. Soc., 130, 1505-1530, 2004.

Vaughan, G., Garland, W. E., Dewey, K. J., and Gerbig, C.: Aircraft measurements of a warm conveyor belt - a case study, J. Atmos. Chem., 46, 117-129, 2003.

Vaughan, G., Methven, J., Anderson, D., Antonescu, B., Baker, L., Baker, T. P., Ballard, S. P., Bower, K. N., Brown, P. R. A., Chagnon, J., Choularton, T. W., Chylik, J., Connolly, P. J., Cook, P. A., Cotton, R. J., Crosier, J., Dearden, C., Dorsey, J. R., Frame, T. H. A., Gallagher, M. W., Goodliff, M., Gray, S. L., Harvey, B. J., Knippertz, P., Lean, H. W., Li, D., Lloyd, G., MartínezAlvarado, O., Nicol, J., Norris, J., Öström, E., Owen, J., Parker, D. J., Plant, R. S., Renfrew, I. A., Roberts, N. M., Rosenberg, P., Rudd, A. C., Schultz, D. M., Taylor, J. P., Trzeciak, T., Tubbs, R., Vance, A. K., van Leeuwen, P. J., Wellpott, A., and Woolley, A.: Cloud Banding and Winds in Intense European Cyclones: Results from the DIAMET Project, Bull. Amer. Meteor. Soc., 96, 249-265, 2015.

Wallace, J. M. and Hobbs, P. V.: Atmospheric Science: An Introductory Survey, Academic Press, 2nd Edn., Amsterdam, 2006.

Wernli, H.: A Lagrangian-based analysis of extratropical cyclones. II: A detailed case-study, Q. J. Roy. Meteor. Soc., 123, 16771706, 1997.

Wernli, H. and Davies, H. C.: A Lagrangian-based analysis of extratropical cyclones. I: The method and some applications, Q. J. Roy. Meteor. Soc., 123, 467-489, 1997.

Whitaker, J. S., Uccellini, L. W., and Brill, K. F.: A Model-Based Diagnostic Study of the Rapid Development Phase of the Presidents's Day Cyclone, Mon. Weather Rev., 116, 2337-2365, 1988.

Wilhelmson, R. B., Jewett, B. F., Shaw, C., Wicker, L. J., Arrott, M., Bushell, C. B., Bajuk, M., Thingvold, J., and Yost, J. B.: A Study of the Evolution of a Numerically Modeled Severe Storm, Int. J. High Perform. C., 4, 20-36, 1990.

Wulfmeyer, V., Behrendt, A., Bauer, H.-S., Kottmeier, C., Corsmeier, U., Blyth, A., Craig, G., Schumann, U., Hagen, M., Crewell, S., Di Girolamo, P., Flamant, C., Miller, M., Montani, A., Mobbs, S., Richard, E., Rotach, M. W., Arpagaus, M., Russchenberg, H., Schlüssel, P., König, M., Gärtner, V., Steinacker, R., Dorninger, M., Turner, D. D., Weckwerth, T., Hense, A., and Simmer, C.: RESEARCH CAMPAIGN: The Convective and Orographically Induced Precipitation Study, Bull. Am. Meteorol. Soc., 89, 1477-1486, 2008. 